RESEARCH REPORT

\title{
Auf neuen Pfaden - Per Anhalter durch die Berylliumchemie
}

\author{
Dominik Naglav, ${ }^{[a]}$ Magnus R. Buchner, ${ }^{[b]}$ Georg Bendt, ${ }^{[a]}$ Florian Kraus*[b] und Stephan \\ Schulz*[a]
}

Keywords: Beryllium; Toxikologie; Glucinium; Sicherheit; Handhabung; Berylliose; Chronische Beryllium Erkrankung CBD

\begin{abstract}
Dieser Bericht soll eine Einführung für alle weniger Erfahrenen auf dem Gebiet der Berylliumchemie darstellen. Aktuelle Forschungsergebnisse über die Giftigkeit des Elements und seiner Verbindungen werden rezensiert und diverse grundlegende und nötige Orientierungshilfen für die sichere und richtige Handhabung in modernen chemischen Forschungslaboren vorgestellt. Phänomenologische Beobachtungen bezüglich der Einzigartigkeit des Elements und seiner Chemie werden mitgeteilt und in den historischen Kontext gestellt.
\end{abstract}

Wir fassen viele Beiträge und Erfahrungen von auf diesem Gebiet leidenschaftlich tätigen Wissenschaftlern zusammen. Es ist uns ein Herzensanliegen, dass unsere Kommilitonen die über Generationen aufgebauten Vorbehalte gegenüber Beryllium und seinen Verbindungen kritisch hinterfragen und überdenken, und so eine neue Ära intensiver Forschung zu diesem verschmähten Element eingeleitet wird.

Wer in aller Welt soll denn das Beryllium und seine Verbindungen sicherer und fachkundiger handhaben können als wir Chemiker? ((Main Text Paragraphs))

* Corresponding Author

Fax: +49 6421 28 - 2575 5; +49 201 183-3830

E-Mail: florian.kraus@chemie.uni-marburg.de; stephan.schulz@uni-due.de

[a] Faculty of Chemistry

University of Duisburg-Essen

Universitätsstraße 7, 45141 Essen

[b] Anorganische Chemie, Fluorchemie

Philipps-Universität Marburg

Hans-Meerwein-Str. Supporting information for this

article is available on the WWW under

http://dx.doi.org/10.1002/zaac.201000xxx or from the author.

\section{E.stroduction}

((Biographical Sketch))

\section{KEINE PANIK!}

Was wissen Sie wirklich über das Beryllium und seine Verbindungen? Was wissen Sie wirklich über deren Giftigkeit? Was wissen Sie wirklich über deren Handhabung und chemischen Eigenschaften? Haben Sie jemals metallisches Beryllium oder seine kristallinen Verbindungen realiter gesehen? Die meisten Chemiker verdanken ihr diesbezügliches Wissen Fachbüchern, Vorlesungen oder, im ungünstigsten Fall, diversen Internet-Foren. Warum aber wird dieses Element von Forschern der beiden letzten Jahrhunderte so sträflich vernachlässigt, ja geradezu gemieden und missachtet? Viele würden nun antworten, dass Beryllium und seine Verbindungen einfach nur Mauerblümchen sind und führen dies dann auf die legendäre Giftigkeit des Berylliums und die damit verbundenen Schwierigkeiten in der Handhabung zurück. Eine andere Antwort könnte sein, dass es heutzutage sehr schwierig geworden ist Beryllium oder irgendeine seiner Verbindungen zu bekommen. Die Gründe hierfür werden später erklärt.

Unserer Ansicht nach ist die gegenwärtige Situation nicht zufriedenstellend und sollte geändert werden. Die vom Element ausgehende Faszination hat bereits aufregende Ergebnisse hervorgebracht, welche im Folgenden kurz zusammengefasst werden. Wir hoffen, dass die Berylliumchemie zukünftig ähnlich intensiv bearbeitet wird, wie dies für seine Nachbarn im Periodensystem in den vergangenen 30 Jahren der Fall war. Das Beryllium ist mehr als eine Laborkuriosität und es darf nicht auf die üblichen Angaben zur Süße mancher seiner Salze, dass es das giftigste, nicht-radioaktive Element sei, ${ }^{[1,2]}$ oder auf die wohlbekannte Verwendung als Fenster für Röntgenstrahlung, reduziert werden. 
Glücklicherweise hat sich in den letzten zwei Dekaden die Einstellung zum Beryllium geändert. Mehrere Wissenschaftler und Forschergruppen untersuchen die Chemie des Berylliums, was an einer recht starken Zunahme an Veröffentlichungen zu präparativen Arbeiten erkannt werden kann. Das Zitat "the number of theoretical investigations published on beryllium chemistry is by far larger than the number of experimental studies" ist jedoch immer noch gültig. Zudem kann "achieving a thorough understanding of beryllium chemistry through the collection of basic knowledge is still the task of chemists." wohl nicht nachdrücklicher formuliert werden. ${ }^{[3]}$

Im Folgenden versuchen wir unser Bestes, um den Einstieg in die Berylliumchemie zu erleichtern. Der stets beim Arbeiten mit so einem vernachlässigten Element in uns wohnende und lebende Pioniergeist (,spiritus praecursoris") möge auf unsere Kampfgefährten übergehen!

Ein Vorwort zur Verwendung von Be und seinen Verbindungen

Das Element zeigt eine Reihe von Eigenschaften, die es für zahlreiche Anwendungen besonders interessant machen: Das Metall ist von sehr geringe Dichte und doch hart, elastisch verformbar und zugleich zugfest - optimal zur Verwendung in der Luft- und Raumfahrt, zum Beispiel im Kreiselkompass oder in Flugstabilisatoren. Berylliumlegierungen können diese mechanischen Eigenschaften noch übertreffen und sind zudem wesentlich korrosionsbeständiger. Solche Legierungen werden in einer Vielzahl von Spezialanwendungen eingesetzt, zum Beispiel für Beschichtungen in Kopfhörern (für wahre Klangenthusiasten), für nichtfunkenreißendes, nicht-ferromagnetisches Werkzeug (Arbeiten in explosionsgefährdeten Bereichen oder an NMR-Spektrometern), und auch für Federn mit hoher Ermüdungsgrenze. Beryllium ist einer der Hauptbestandteile der Spiegel von Weltraumteleskopen, zum Beispiel des James-Webb-Weltraumteleskops. ${ }^{[4 a), b)]}$

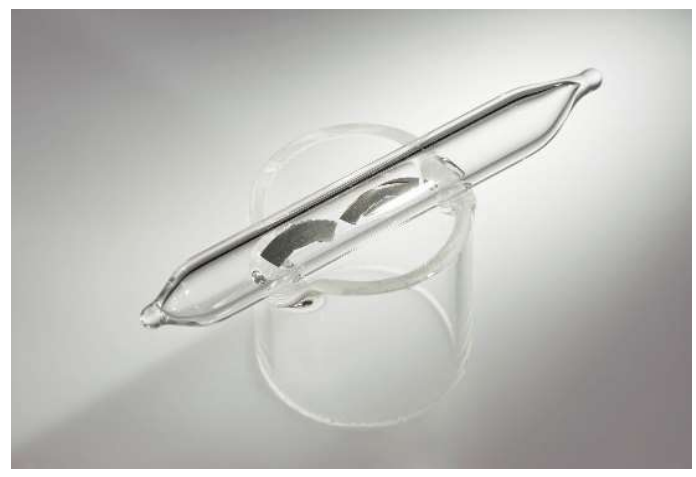

Abbildung 1. Eine Probe reinen Be-Metalls in einer unter Ar abgeschmolzenen Glasampulle.

\footnotetext{
Was hat es mit der ,Giftigkeit" von Beryllium auf sich?
}

Zunächst die Statistik: Im Jahr 2013 wurden bei der Deutschen Gesetzlichen Unfallversicherung (DGUV) 21 Verdachtsfälle einer Berufskrankheit im Zusammenhang mit Beryllium verzeichnet. ${ }^{[5]}$ Davon wurden drei Fälle bestätigt und als Berufserkrankung anerkannt. Aus der Statistik der DGUV für das Jahr 2013 ist weiterhin ersichtlich, dass seit 2010 dreizehn Fälle von Berylliose und zwei assoziierte Todesfälle aufgetreten sind. ${ }^{[6]}$ Im gleichen Zeitraum wurden 39 anerkannte Erkrankungen und 52 Todesfälle im Zusammenhang mit Benzol anerkannt, bei der Silikose waren es schrecklicherweise sogar 4232 anerkannte Erkrankungen und 1469 Sterbefälle. Um diese Zahlen nun einzuordnen: 17430 Personen waren im Jahr 2006, gemäß der DGUV, im Kontakt mit $\mathrm{Be}$ und seinen Verbindungen, davon haben $0,07 \%$ eine Berylliose entwickelt und $0,0004 \%$ sind daran leider verstorben. ${ }^{[7]} \mathrm{Im}$ Jahr 2012 waren in Deutschland 564927 Personen Asbest ausgesetzt, von denen 0,75\% eine Silikose entwickelten und $0,26 \%$ verstarben. ${ }^{[8]}$ Zieht man nun nur die Industriezweige zum Vergleich heran, bei denen mit großer Exposition gegenüber Beryllium zu rechnen ist, also beim Schmelzen, Gießen und Verarbeiten des Metalls und seiner Legierungen (662 Arbeiter im Jahr 2011, 1,96\% Berylliose, 0,30\% Todesfälle), so erscheint das Risiko mit dem von Asbest vergleichbar. ${ }^{[7]}$ Wir möchten uns für die uneinheitlichen Jahreszahlen der Statistiken entschuldigen, uns waren auch nach langer Recherche keine anderen zugänglich. Die mit Prävention befassten Stellen sehen die Exposition gegenüber Beryllium im Rahmen von Forschung und Entwicklung generell als niedrig an. ${ }^{[7]}$

Aktuelle medizinische Studien legen nahe, dass eine allergische Reaktion, sowohl für die akute als auch für die chronische Berylliose (chronic beryllium disease CBD) verantwortlich ist. Die Untersuchungen weisen auch darauf hin, dass Be-Metall selbst nicht akut toxisch ist und dass die Humankanzerogenität nicht zweifelsfrei belegt ist. ${ }^{[6,9-17]}$ Strupp und Mitarbeiter schlagen zudem vor, dass das Beryllium und seine Verbindungen nicht als akut toxisch (Totenkopfsymbol) eingestuft werden sollten, außer die Toxizität wird vom Anion induziert. ${ }^{[15 a)-}$ c)] An dieser Stelle ist anzumerken, dass die Strupp'schen Arbeiten vom REACH Beryllium Consortium, einem Industrieverband unter Führung von Materion Brush, dem größten Be-Produzenten der USA, finanziert wurden. ${ }^{[14]}$ Tatsächlich scheint die „Giftigkeit" von Beryllium und seinen Verbindungen irgendwo auf der Grenze zwischen einer allergischen Überempfindlichkeitsreaktion und einer Autoimmunreaktion zu liegen. ${ }^{[18]}$ Gemäß dem Global harmonisierten System zur Einstufung und Kennzeichnung von Chemikalien (GHS) wird ein Stoff mit dem Gefahrensymbol ,giftig“ (Totenkopf) versehen, wenn gewisse Kriterien erfüllt sind (Tabelle 1). Andernfalls ist die Verbindung als nicht akut toxisch einzustufen und darf kein Totenkopfsymbol tragen. In Tabelle 2 sind einige $\mathrm{LD}_{50}$-Werte von Berylliumverbindungen zusammengetragen. Manche von ihnen tragen das Totenkopfsymbol für akute Toxizität. Eine „Vergiftung“ mit Beryllium und seinen Verbindungen kann in ihrer ,akuten“ Form verlaufen, wenn hohe Dosen in einer kurzen Expositionszeit aufgenommen wurden. Sie kann in ihrer „chronischen“ 
Form verlaufen, wenn niedrige Dosen über lange Zeiträume aufgenommen wurden. Kürzlich erschienene molekularbiologische Untersuchungen schlagen vor, dass die körperlichen Reaktionen wahrscheinlich auf eine Überreaktion der Immunantwort zurückzuführen sind, was durch die Existenz von auf berylliumhaltige Spezies sensitivierten T-Zellen indiziert ist. Solche T-Zellen konnten sowohl in Berylliosepatienten, als auch in Tierversuchen und Zellexperimenten nachgewiesen werden. $^{[19]}$ Die spezifische Immunantwort wird wahrscheinlich durch das HLA-DP-Protein, welches ein Teil des Protein/Peptid-Antigenrezeptors auf der Oberfläche von T-Zellen ist, ausgelöst, das eine hohe Affinität gegenüber bisher unbekannten Berylliumverbindungen hat. ${ }^{[18,20,21]}$ Mutationsexperimente an HLA-DP implizieren, dass die Be-Spezies an lediglich einige wenige Aminosäuren von Seitenketten ${ }^{[22,23]}$ oder an nur eine spezifische Glutaminsäure des Proteins gebunden ist. $^{[11]}$

Tabelle 1. LD $D_{50}$-Kriterien für das GHS-Zeichen „giftig“ (Totenkopf). ${ }^{[24]}$

\begin{tabular}{|c|c|}
\hline Expositionsweg & $\mathrm{LD}_{50}$ (Ratte) \\
\hline Oral & $\leq 300 \mathrm{mg} / \mathrm{kg}$ Körpergewicht \\
\hline Dermal & $\leq 1000 \mathrm{mg} / \mathrm{kg}$ Körpergewicht \\
\hline Gase & $\leq 2500 \mathrm{ppm}$ \\
\hline Dämpfe & $\leq 10 \mathrm{mg} / 1$ \\
\hline Stäube und Nebel & $\leq 1 \mathrm{mg} / 1$ \\
\hline
\end{tabular}

Verschiedene Arbeitsgruppen schlagen vor, dass die bevorzugte Komplexierung des $\mathrm{Be}^{2+}$-Kations in Proteinen mit dem Austausch von Protonen in starken Wasserstoffbrückenbindungen zwischen Carboxyl- und Hydroxylgruppen in Aminosäureseitenketten, wie in Schema 1 gezeigt, zu begründen ist. ${ }^{[2,25,26]}$ Diese starken Wasserstoffbrückenbindungen wurden über ihre ausgeprägte Tieffeldverschiebung im ${ }^{1} \mathrm{H}-\mathrm{NMR}$-Spektrum im Bereich von $10-22 \mathrm{ppm}$ nachgewiesen. ${ }^{[25]}$ Ersetzt man ein zweifach-koordiniertes Proton einer Wasserstoffbrückenbindung mit einem Beryllium-Kation, welches eine starke Präferenz zu einer tetraederähnlichen Koordination zeigt, so würde der Bindungswinkel der vormaligen Wasserstoffbrückenbindung drastisch auf circa $109^{\circ}$ reduziert. Der Bindungswinkel von Wasserstoffbrückenbindungen, insbesondere von starken, weicht üblicherweise nur wenig von der Linearität ab und wird fast nie unterhalb von $120^{\circ}$ beobachtet. Diese strukturelle Deformation sollte also $\mathrm{zu}$ großen Konformationsänderungen im Protein führen. Es ist jedoch fraglich, ob eine $\mathrm{Be}^{2+}$-Spezies (ein ,nacktes“ $\mathrm{Be}^{2+}$ Kation ist unter physiologischen Bedingungen höchst unwahrscheinlich) das Proton einer OH-Funktion durch Bindung an das O-Atom so acidifizieren könnte, dass es freigesetzt wird - dies wurde unserem Wissen nach jedoch bisher nicht gezeigt.

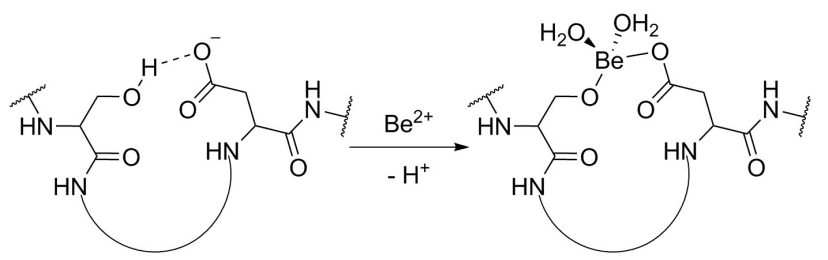

Schema 1. Vorgeschlagener Interaktionsmechanismus von $\mathrm{Be}^{2+}$ mit Proteinen. ${ }^{[10,25,26]}$

Weiterhin zeigen biochemische Untersuchungen, dass Proteine mit mehreren Berylliumkationen beladen werden können. ${ }^{[25,27]}$ Die Bindung mehrerer Berylliumkationen an ein Protein könnte für die akute Berylliose von Relevanz sein, aber für das Verständnis der chronischen Berylliose ist dieser Befund wohl nicht brauchbar, da bereits geringste Mengen an Beryllium $\left(2 \mu \mathrm{g} / \mathrm{m}^{3}\right)$ und seiner Verbindungen in der Lunge höchst ,giftig“ wirken können, obwohl die Löslichkeit von Berylliumverbindungen unter physiologischen Bedingungen in der Lunge vergleichsweise klein ist. ${ }^{[3,28]}$ Es sollte angemerkt werden, dass eine Verbindung, welche die Alveolen in der Lunge erreichen soll, nur gering wasserlöslich sein darf, ansonsten wird sie bereits im oberen Atemtrakt gebunden. Zudem spielt auch die Partikelgröße eine Rolle. Aus diesen Gründen erwartet man lediglich eine ganz geringe Konzentration an Beryllium in den Zellen. Vermutlich rührt die nicht vorhersagbar lange Latenzzeit von BerylliumLungenerkrankungen (es wurden Latenzzeiten von bis zu 30 Jahren berichtet, ein Maximum an Erkrankungen wird aber bereits nach drei Jahren beobachtet) ${ }^{[29]}$ von einer spezifischen Interaktion im HLA-DP-T-ZellRezeptorkomplex her. Die Berylliose als Lungenerkrankung muss also ähnlich wie die Silikose, die Asbestose und am meisten wohl wie die Sarkoidose eingestuft werden. Bei letzterer Erkrankung werden in der Lunge Fremdkörper unter Narbenbildung des Lungengewebes eingekapselt, welches dann seine Effizienz für die $\mathrm{O}_{2}$-Aufnahme und $\mathrm{CO}_{2}$-Abgabe weitgehend einbüßt. Die Ähnlichkeit zwischen Berylliose und Sarkoidose, deren Ursachen heute noch unklar sind, ist daher unbedingt zu betonen. ${ }^{[15 a)]}$

Man nimmt an, dass nur 1-5\% der Allgemeinbevölkerung eine genetische Prädisposition für die chronische Berylliose aufweisen. ${ }^{[30 \mathrm{a}), \mathrm{b})]}$ Die eindeutige Diagnose der chronischen Verlaufsform verlangt eine Unterscheidung von der Sarkoidose, welche eine klinisch ähnliche, granulomatöse Lungenerkrankung ist. Ein wichtiges Kriterium hierfür ist der Nachweis, dass eine Exposition gegenüber $\mathrm{Be}$ und seinen Verbindungen vorgelegen hat. ${ }^{[31 \mathrm{a}), \mathrm{b})]}$ Man schätzte, dass Konzentrationen oberhalb von $0.02 \mu \mathrm{g} / \mathrm{m}^{3}$ nötig sind, um die chronische Berylliumerkrankung auszubilden. ${ }^{[30-33]}$ Aus der verfügbaren medizinischen Literatur wurde abgeleitet, dass $\mathrm{Be}$ und seine Verbindungen als krebserregend eingestuft werden sollten. ${ }^{[32]}$ An zwei verschiedenen Versuchstierspezies wurden Inhalationsstudien durchgeführt, welche eine signifikante Steigerung der Lungenkrebsraten zeigten. ${ }^{[30]}$ Allerdings sind manche Expertengremien der Ansicht, dass die Studien an beruflich exponierten Menschen nicht hinreichend sind, um die Kanzerogenität beim Menschen zu beweisen. ${ }^{[33]}$ 
Da die biochemischen Mechanismen, welche $\mathrm{zu}$ Berylliumkrankheiten führen, noch immer unbekannt sind, raten wir zur Vorsicht und empfehlen besondere (Sicherheits-) und Vorsichtsmaßnahmen (s.u.) für den Umgang mit Beryllium und seinen Verbindungen.

\section{Was ist an Gesetzen und medizinischen Vorgaben $\mathrm{zu}$ beachten, wenn man mit Be und seinen Verbindungen arbeiten möchte?}

Wir erörtern die Situation hauptsächlich für Deutschland, und kurz für die USA, das UK und Russland, im Falle anderer Nationen können die benötigten Informationen von den Landesgesundheitsorganisationen erhalten werden.

Wenn man vermutet, dass der Arbeitsplatzgrenzwert (AGW) einer Be-Verbindung überschritten werden könnte, dürfen Mitarbeiter nur dann mit dem Stoff arbeiten, nachdem sie sich beim Betriebsarzt vorgestellt haben. ${ }^{[34 a)-c)] ~ D a n n ~ s i n d ~ d e n ~ B e s c h a ̈ f t i g t e n ~ a u c h ~}$ regelmäßige Untersuchungen beim Betriebsarzt anzubieten. Da das Be und seine Verbindungen als Humankarzinogen Kategorie 1B eingestuft sind, sind die Technischen Regeln für Gefahrstoffe TRGS $410 \mathrm{zu}$ befolgen, welche detailliert auf notwendige Maßnahmen eingehen. ${ }^{[35]}$ Da jedoch Be und seine Verbindungen als krebserregend eingestuft sind, gibt es derzeit noch keinen Arbeitsplatzgrenzwert, ${ }^{[29]}$ was den einleitenden Satz ad Absurdum führt. Kürzlich wurde für Deutschland ein AGW von $0,060 \mu \mathrm{g} / \mathrm{m}^{3}$ vorgeschlagen. ${ }^{[36]}$ Wir merken an, dass der Tabak einer Zigarette bis zu 0,74 $\mu \mathrm{g}$ Be, der Rauch bis $\mathrm{zu} 0,074 \mu \mathrm{g}$ pro Zigarette enthalten kann. ${ }^{[37]}$ Dies bringt uns zu der nicht gerade neuen Erkenntnis, dass Rauchen ziemlich ungesund ist. Ist man also Raucher, oder beschäftigt man etwa gar nur Passivraucher im Labor, dann wird die moderne instrumentelle Analytik auch Beryllium in der Luft nachweisen können - und das sogar in Bereichen, in denen garantiert noch nie mit Beryllium oder Berylliumverbindungen gearbeitet wurde (auf dem Gang, im Treppenhaus, ...). In den Vereinigten Staaten von Amerika schlägt die OSHA eine Absenkung des Acht-Stunden-Expositionslimits von 2,0 $\mu \mathrm{g} / \mathrm{m}^{3}$ auf $0,2 \mu \mathrm{g} / \mathrm{m}^{3}$ vor. $^{[38]}$ Die gegenwärtigen ,threshold limit values" (TLV) der USA können hier abgerufen werden (https://www.osha.gov/dts/chemicalsampling/data/CH_22 0600.html). Zudem sind die Maßgaben des Titels ${ }^{-}$10, Code of Federal Regulations, "Energy", Chapter 3, "Department of Energy", Part 850, "Chronic Beryllium Disease Prevention Program" "[39] und Titel 29, Code of Federal Regulations, "Labor", Chapter 17, "Occupational Safety and Health Administration, Department of Labor", Part 1910, "Occupational Safety and Health Standards"[40-42] für die USA zu beachten. ${ }^{[43]}$ Im Vereinigten Königreich greifen die Richtlinien des "Control of Substances Hazardous to Health" (COSHH) [44] und in Russland die GOST $12.1995-76 .^{[45,46]} \mathrm{Die}$ Grenzwerte dieser Länder, und der meisten Länder der EU, liegen derzeit im Bereich von 2 bis $0,2 \mu \mathrm{g} / \mathrm{m}^{3}$. ${ }^{[7]}$
Keine deutsche Regel scheint derzeit wirklich passend $\mathrm{zu}$ sein, daher raten wir zu einem engen Kontakt zur Betriebsärztin. Sie ist der Situation gewahr und wird auch künftige Entwicklungen verfolgen, um uns Chemikern mit Rat zur Seite zu stehen, zum Beispiel bei der Einstufung, ob man im Labor mit einer hohen Exposition $\mathrm{zu}$ rechnen hat (was nicht der Fall ist). Beispiele von Arbeiten mit hohem Expositionsrisiko sind bestens bekannt. $^{[29]}$ Für den Betriebsarzt müssen wir dies abschätzen, jedoch kommt auch hier die Literaturrecherche zur Hilfe: Lege artis durchgeführte chemische Laborarbeiten sind nicht als hohes Expositionsrisiko eingestuft (man sollte sich immer in Erinnerung rufen, dass Vorschriften zur Arbeitssicherheit hauptsächlich auf Industriearbeiter abzielen, welche mit großen Mengen an Gefahrstoffen umgehen). Außerdem enthalten die Regelwerke auch Arbeitssituationen, bei denen von keiner Exposition ausgegangen werden kann das Arbeiten in geschlossenen Apparaturen, was man als Chemiker ja typischerweise immer macht - gehört dazu. Die Probennahme hat aber meistens zur Folge, dass die geschlossenen Bedingungen nicht aufrecht erhalten werden können. Für solche Fälle geben wir unten Empfehlungen.

Die Bundesanstalt für Arbeitsschutz und Arbeitsmedizin, BAuA, fordert in ihren Technischen Regeln für Gefahrstoffe, TRGS, dass man Aufzeichnungen darüber führt, welche das Expositionsmaß und die Dauer der Tätigkeiten mit krebserregenden Stoffen enthalten (TRGS 410). ${ }^{[34]}$ Das Beryllium und seine Verbindungen fallen natürlich darunter, insbesondere da für den Umgang Atemschutz und Handschuhe empfohlen sind. Führt man jedoch die Arbeiten im Forschungslabor in geschlossenen Apparaturen durch und nur in Mengen, die nach der TRGS 526 als „laborüblich“ bezeichnet werden, dann sind diese Aufzeichnungen nicht nötig. ${ }^{[29]}$ Auch wenn das erste Kriterium sich im Labor nicht streng einhalten lässt (Probennahme), sind die Mengen klein und die Expositionszeiten sehr kurz, so dass keine Aufzeichnungspflicht besteht. Die Laborrichtlinie TRGS 526 befreit die Laborchemiker sozusagen von der TRGS 410 (und von vielen anderen Regelwerken) - aber das auch nur, wenn man sich an die Laborrichtlinie hält! ${ }^{[35]}$

Aus den vorhergegangenen Absätzen kann man schließen, dass die akute Berylliose, also eine akute Vergiftung, ein in hohem Maße unwahrscheinlicher Fall für den Chemiker ist. Folgende Tabelle 2 enthält die $\mathrm{LD}_{50}-$ Werte einiger Verbindungen zum Vergleich. ${ }^{[30-33]}$

Table 2. LD50-Werte verschiedener Verbindungen. Das Kristallwasser der Verbindungen wurde bei den Formeln weggelassen.

\begin{tabular}{|c|c|}
\hline Verbindung & LD $_{\mathbf{5 0}}$ oral (Spezies) \\
\hline $\mathrm{Be}$ & Nicht verfügbar \\
\hline $\mathrm{Be}(\mathrm{OAc})_{2}$ & Nicht verfügbar \\
\hline
\end{tabular}




\begin{tabular}{|c|c|}
\hline $\mathrm{BeBr}_{2}$ & Nicht verfügbar \\
\hline $\mathrm{BeCl}_{2}$ & $86 \mathrm{mg} / \mathrm{kg}$ (Ratte) \\
\hline $\mathrm{BeF}_{2}$ & $98 \mathrm{mg} / \mathrm{kg}$ (Ratte) \\
\hline $\mathrm{Be}\left(\mathrm{NO}_{3}\right)_{2}$ & Nicht verfügbar \\
\hline $\mathrm{BeO}$ & Nicht verfügbar \\
\hline $\mathrm{BeSO}$ & $82 \mathrm{mg} / \mathrm{kg}$ (Ratte) \\
\hline $\mathrm{KCN}$ & $5 \mathrm{mg} / \mathrm{kg}$ (Ratte) \\
\hline $\mathrm{NaN}_{3}$ & $27 \mathrm{mg} / \mathrm{kg}$ (Ratte) \\
\hline $\mathrm{Hg}\left(\mathrm{NO}_{3}\right)_{2}$ & $26 \mathrm{mg} / \mathrm{kg}$ (Ratte) \\
\hline
\end{tabular}

Für den Menschen sind keine Angaben verfügbar. In Tierversuchen konnte gezeigt werden, dass schlechtlösliche Be-Verbindungen weniger giftig als gut lösliche sind. Die $\mathrm{LD}_{50}$-Werte liegen, bezogen auf den Be-Gehalt, zwischen $10-20 \mathrm{mg} / \mathrm{kg}(1,1-2,2 \mathrm{mmol} / \mathrm{kg})$ (Ratte), was ihre Giftigkeit zeigt. Allerdings liegen bisher keine Details über die Symptome der Vergiftung und die Zielorgane vor. ${ }^{[30-33]}$ Zudem hält nun die Aussage, dass $\mathrm{Be}$ das giftigste, nicht-radioaktive Element ist, der kritischen Überprüfung nicht stand. Die Giftigkeit von Be-Verbindungen kann am besten mit der Giftigkeit löslicher Hg- oder As-Verbindungen verglichen werden und damit gehen Chemiker seit ein paar hundert Jahren ja erfolgreich um, ohne sich dauernd akut zu vergiften. Es sollte zudem angemerkt werden, dass chemische Waffen wenigstens eine Größenordnung giftiger (bezogen auf die $\mathrm{LD}_{50}$ ) als KCN sind, und doch wurden solche Verbindungen bedauerlicherweise sogar im industriellen Maßstab hergestellt - die Handhabung von extrem toxischen Verbindungen ist also für Chemiker und Chemieingenieure seit Jahrzehnten kein Problem.

\section{Wie erhält man Be und seine Verbindungen?}

Neben Sicherheitsbedenken aufgrund des toxikologischen oder allergenen Potentials von Beryllium und seinen Verbindungen ist die Beschaffung des reinen Elements problematisch, da es in Kernwaffen und Kernreaktoren als Neutronenreflektor Anwendung findet. Der Handel mit Be-Metall wird von nationalen und internationalen Kernenergiebehörden streng reglementiert und somit ist die Zahl von Laborhändlern sehr klein. ${ }^{[4]}$

"The current status of the beryllium industry is unclear because of beryllium uses in nuclear weapons and the secret nature of this business." - Beryllium chemistry and processing von Kenneth A. Walsh ${ }^{[4]}$

Auch für industrielle Zwecke (Be-Legierungen und so weiter) gibt es nur wenige Anbieter, von denen Materion (USA) der größte, gefolgt vom zweitgrößten Anbieter NGK (JPN), ist. Auch die „Ulba Metallurgical Plant“ in Kasachstan ist ein Erzeuger von Be-Metall und seinen Legierungen. ${ }^{[4]}$

Nach unserem Kenntnisstand ist es in Deutschland unmöglich, metallisches Beryllium zu kaufen. Im Kontakt mit mehreren gut etablierten Laborhändlern (SigmaAldrich, Alfa Aesar, abcr, Chempur, Strem, smartelements) wurde uns immer nur mitgeteilt, dass der bürokratische Aufwand in puncto
Sicherheitsvorkehrungen zu groß im Verhältnis zur Zahl der möglichen Kunden sei. Soweit uns bekannt, kann man nur $\mathrm{BeCl}_{2}$ (25 g für $2445 €$ - Sigma Aldrich 16.6.2015), $\mathrm{BeSO}_{4} \cdot 4 \mathrm{H}_{2} \mathrm{O}$ und $\mathrm{BeO}$ in Deutschland erwerben. Für andere Länder mag es Versorger und Laborhändler geben (http://www.goodfellow.com/).

Da weder $\mathrm{BeO}$ noch $\mathrm{BeSO}_{4} \cdot 4 \mathrm{H}_{2} \mathrm{O}$ optimale Edukte für metallorganische oder koordinationschemische Umsetzungen sind, und da der Preis für wasserfreies Berylliumchlorid geradezu unverschämt hoch ist, sind andere Zugangsmöglichkeiten $\mathrm{zu}$ erschließen, um die gewünschten Reagenzien zu erhalten. Ein guter Start wäre ein Zugang zu Be-Metall, welches bei höheren Temperaturen mit den Halogenen direkt $\mathrm{zu}$ den Berylliumhalogeniden $\mathrm{BeX}_{2}(\mathrm{X}=\mathrm{Cl}, \mathrm{Br}, \mathrm{I})$ kontrolliert reagiert (Schema 2). Lediglich das Fluorid wird am besten nicht auf diesem Wege hergestellt, da elementares Fluor zu reaktiv ist. ${ }^{[47]}$ Woher soll man nun metallisches Beryllium bekommen, wenn es keine Händler gibt? Die Lösung ist leicht: Man fragt in der chemischen oder physikalischen Fakultät nach Be-Proben, welche oft noch in dunklen Lagern völlig unangerührt vorhanden sind. Dies mag zwar keine wirkliche Versorgung sein, aber es ist ein Anfang. Zudem sind die Befragten oft sehr froh das Beryllium endlich los zu werden. Man bekommt zwar oft nur wenig, aber wenn man es nicht versucht, bekommt man gar nichts. Ein weiterer guter Weg ist das Aufbrechen alter Röntgenröhren, die der Kristallograph des Vertrauens auch gerne abgibt. Eine detaillierte Anleitung, wie man solche unter Hochvakuum stehenden Röntgenröhren aufbricht und das Be-Metall extrahiert, findet sich im Anhang. Man kann natürlich auch versuchen, eine der oben stehenden Firmen anzusprechen und auf positives Feedback zu hoffen. Hat man einmal Zugang zu Beryllium, so kann mit den Umsetzungen zu den Wunschverbindungen begonnen werden, bei welchen es sich meist um die oben genannten Halogenide handeln dürfte. In den meisten Fällen ist die fraktionierende Sublimation zur Aufreinigung der Halogenide voll und ganz ausreichend, um Verunreinigungen wie Fe(III) usw. $\mathrm{zu}$ entfernen. ${ }^{[47]}$ Eine ausführliche Anleitung findet sich im Abschnitt „Wie geht man mit Be und seinen Verbindungen um?"“, sowie im Anhang. Nachdem man die Edukte hergestellt hat ist man für die eigene BeChemie bestens gerüstet.

$$
\begin{array}{ccc}
\left(\mathrm{NH}_{4}\right)_{2}\left[\mathrm{BeF}_{4}\right] & \stackrel{>500{ }^{\circ} \mathrm{C}}{\longrightarrow} & \mathrm{BeF}_{2}+2 \mathrm{NH}_{4} \mathrm{~F} \\
\mathrm{Be}+\mathrm{Cl}_{2} & \stackrel{700{ }^{\circ} \mathrm{C}}{\longrightarrow} & \mathrm{BeCl}_{2} \\
\mathrm{Be}+\mathrm{Br}_{2} & \stackrel{550{ }^{\circ} \mathrm{C}}{\longrightarrow} & \mathrm{BeBr}_{2} \\
\mathrm{Be}+\mathrm{I}_{2} & \stackrel{480{ }^{\circ} \mathrm{C}}{\longrightarrow} & \mathrm{BeI}_{2} \\
\mathrm{Be}(\mathrm{OH})_{2}+2 \mathrm{HF} & \stackrel{\text { evap. several times }}{\longrightarrow} & \mathrm{BeF}_{2}+2 \mathrm{H}_{2} \mathrm{O}
\end{array}
$$

Schema 2. Einige Synthesebedingungen von Be-Halogeniden ${ }^{[4]}$

Wie geht man mit Be und seinen Verbindungen um? 
Im Allgemeinen sind zwei Aspekte für den Umgang mit $\mathrm{Be}$ und seinen Verbindungen $\mathrm{zu}$ beachten: Der eine bezieht sich auf die Sicherheitsvorkehrungen, um einen direkten Kontakt zu vermeiden, der andere das Wissen über die besonderen Handhabungstechniken solcher Verbindungen in der modernen, fortgeschrittenen anorganischen Chemie.

Kontakt und Inkorporation von Beryllium und seinen Verbindungen müssen beim Arbeiten ausgeschlossen werden. Der lege artis arbeitende Laborchemiker sieht sich lediglich mit der Inhalation als mögliche Expositionsroute konfrontiert. Ein Verschlucken kann nur absichtlich erfolgen. Ein jeder Chemiker weiß aus seinen Vorlesungen, dass das toxikologische Potential einer Verbindung auf dem direkten Kontakt mit ihr basiert. Wird der Kontakt vermieden, so ist die giftige Verbindung so gefährlich (oder ungefährlich) wie jeder andere Stoff! Beim Kontakt spielt dann sowohl die Konzentration, als auch die Expositionsdauer eine Rolle was ja schon Paracelsus im Jahr 1538 festgestellt hat.

Also sollte man sich an die Regeln halten, die für den Umgang mit noch nicht eingestuften Stoffen empfohlen werden, welche jeder aus Sicherheitsbelehrungen sowie der eigenen Ausbildung zum Chemiker kennt. Zusätzlich seien die folgenden Methoden empfohlen:

Technische Schutzmaßnahmen und Anforderungen an die Infrastruktur: Eine grundlegende Anforderung ist ein (eigenes) gut ziehendes Digestorium, in dem man mit Beryllium und seinen Verbindungen immer arbeitet, um die Exposition mit Be-haltigen Stäuben oder Aerosolen auszuschließen. Um überhaupt eine Betriebselaubnis $\mathrm{zu}$ bekommen, müssen moderne Laboratorien diese Anforderung selbstverständlich erfüllen. Somit sind, was den regelmäßig zu prüfenden und geprüften Abzug angeht, keine besonderen Maßnahmen erforderlich, außer dass an ihm ein Warnschild angebracht wird, elches darüber informiert, dass in ihm mit krebserregenden Stoffen (Be und Verbindungen) gearbeitet wird. Auch die Labortüren können so beschriftet werden, wenn man das ganze Labor für den Umgang mit krebserregenden Stoffen ausweist. Wir merken hier zudem an, dass ein zugelassenes und regelmäßig geprüftes Digestorium garantieren sollte (wir kennen alle die Situation der Laborlüftungen), dass unter normalen Betriebsbedingungen keine Gefahrstoffe austreten. Ein Unfall ist jedoch keine normale Betriebsbedingung, so dass man dann unter Umständen nicht vom Abzug geschützt ist. Die Handhabung von flüchtigen oder staubenden Stoffen sollte im Allgemeinen immer im Abzug und unter Schutzgas (geschlossene Apparatur) erfolgen (doppelter Schutz: Abzug + geschlossene Apparatur), da im Falle der stark hygroskopischen Berylliumhalogenide der Luftkontakt aufgrund der vorhandenen Feuchtigkeit ausgeschlossen werden muss. Wir empfehlen den Einsatz der Schlenk-Technik, der damit verbundenen Schutzgasanlagen, sowie den Einsatz von Handschuhkästen. Cave: Das Arbeiten mit der Schlenk-Technik ist immer ,inerter“ als das Arbeiten an einem Handschuhkasten, man denke nur an die Größe der beteiligten Oberflächen und daran, dass der
Handschuhkasten ja nicht ausgeheizt werden kann. Eine Übersicht der von uns typischerweise eingesetzten Glasgerätschaften, Digestorien und Handschuhkästen findet sich im Anhang. Unglücklicherweise muss ein Forscher meist sein Labor und seine Handschuhkästen mit anderen teilen, so dass mit besonderem Nachdruck auf die Sicherheit der anderen geachtet werden muss, was sich durch organisatorische Sicherheitsmaßnahmen (s.u.) bewerkstelligen lässt. Jeder, der mit im Labor oder am Handschuhkasten arbeitet, muss gesondert sicherheitsbelehrt werden, um die gesundheitlichen Aspekte von Beryllium und seinen Verbindungen und den nötigen Sicherheitsvorkehrungen verstanden $\mathrm{zu}$ haben. Dieser Artikel stellt dafür eine Basis dar. Jeder im Labor und jedes Mitglied der Arbeitsgruppe soll von den möglichen Gefahren beim Umgang mit $\mathrm{Be}$ und seinen Verbindungen Bescheid wissen. Glasgeräte oder andere Laboreinrichtungen sollte man nicht teilen, um eine Kontamination auszuschließen (eine Dekontamination hängt vom Stoff $\mathrm{ab}$, bei Be und seinen Verbindungen empfiehlt sich meist verdünnte $\mathrm{HCl}$ ). Häufig wird man sich Glasgeräte teilen müssen, daher ist unbedingt auf deren Sauberkeit vor der Weitergabe zu achten. Alle Beenthaltenden Gerätschaften, der Abfall oder die persönliche Schutzausrüstung wie zum Beispiel mehrfach verwendbare Handschuhe, müssen im Abzug oder einem abgesaugten Schrank bis zur Reinigung oder Entsorgung aufbewahrt werden, keinesfalls wird etwas auf dem Labortisch abgestellt oder gehandhabt! Der Laborkittel soll getrennt von den Laborkitteln der anderen Mitarbeiter aufbewahrt werden. Ohne die Verwendung einer bruchsicheren Umverpackung werden keine Beenthaltenden (Glas)-Gerätschaften aus dem Labor gebracht, damit beim Transport auch wirklich nichts passieren kann. Wir empfehlen die Verwendung von Metalleimern mit abschließbaren Deckeln und mit Einsätzen aus Styropor für den Transport von beispielsweise Schlenkrohren, NMR-Röhrchen, etc. für den Transport zum Handschuhkasten, zum NMRSpektrometer, ...

Organisatorische Schutzmaßnahmen: Mitarbeiter, Laborkollegen und Besucher müssen auf die (erwiesenen und vermuteten) Gefahren von $\mathrm{Be}$ und seinen Verbindungen hingewiesen werden. Wir empfehlen außerdem, dass jeder Zugang, der zu einem Arbeitsbereich mit Be führt, klar und deutlich mit Warnschildern gekennzeichnet wird, am besten so, dass das Labor nicht betreten werden kann, ohne dass man die Warnzeichen wegklappen muss. Eine Anbringung über den äußeren Türklinken, so dass diese verdeckt ist, hat sich bewährt. Die Warnschilder für einen Arbeitsbereich mit Be sollten diese (für die EU) offiziellen Schilder enthalten:

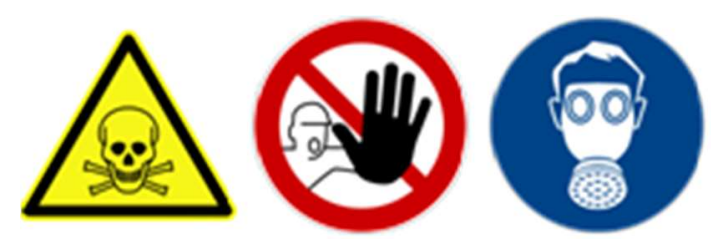

Abbildung 2. Warnschilder (für die Europäische Union) 
Persönliche Schutzmaßnahmen: Beim Arbeiten mit Be und seinen Verbindungen sollte immer eine Atemschutzmaske getragen werden. Diese muss in der Lage sein kleine Partikel auszufiltern, um eine Inhalation $\mathrm{zu}$ vermeiden. Solche Staubfilter werden in drei Schutzstufen angeboten, von FFP-1 bis FFP-3, wobei letztere auch kleinste Partikel bis $0.6 \mu \mathrm{m}$ abtrennt. Eine ausführliche Beschreibung des betrieblichen Atemschutzes und die zuvor und dabei notwendigen Maßnahmen sind von der DGUV verfügbar, siehe die Veröffentlichung BGR/GUV-R 190. ${ }^{[48]}$ Wir empfehlen für alle Routinearbeiten die ausschließliche Verwendung von FFP3-Filtermasken (zum Beispiel von 3M). Beim Umgang mit Beryllium und den Halogenen kann eine Vollmaske mit Gas- und Partikelfilter (ABEK2P3/A2B2E2K2-P3) eingesetzt werden - aber würde man im Labor wirklich eine "Gasmaske“ aufsetzen, nur weil man mit den Halogenen arbeitet? Wohl nicht, da man ja einen funktionieren Abzug und eine geschlossene Apparatur verwendet. Also empfehlen wir wieder ,nur“ eine FFP-3-Partikelfiltermaske (diese haben einen sehr geringen Atemwiderstand, so dass nur eine Angebotsuntersuchung beim Betriebsarzt erfolgen kann). Im Falle des Arbeitens mit flüchtigen, metallorganischen Verbindungen oder mit Verbindungen, die nicht unmittelbar bei Luftkontakt hydrolysieren, setzen wir Halbmasken mit Aktivkohlefiltern ein (ABEKP3-Filter, zum Beispiel die MSA Advantage 400). Es muss an dieser Stelle angemerkt werden, dass die Verwendung von Partikelfiltermasken, Halbmasken oder Vollmasken den vorherigen Besuch beim Betriebsarzt erfordern kann bzw. erfordert. Näheres hierzu regelt die Veröffentlichung der DGUV BGI/GUV-I 504-26. . ${ }^{[50]}$ Für den Schutz der Hände werden Standardprodukte (Latex, Nitril, usw.) eingesetzt, abhängig von den verwendeten Solventien. Schutzbrille und Laborkittel sind ohnehin Pflicht.

Entsorgung von Be-haltigen Abfällen: Beim Arbeiten mit Beryllium werden immer Reste und Abfälle anfallen welche fachmännisch und sicher entsorgt werden müssen. Für herkömmlichen Laborabfall, wie Wischtücher, Einweghandschuhe, Filterpapiere, empfehlen wir die Benutzung eines eigenen Entsorgungsbehälters zusätzlich zu den Standardabfallbehältern für den ,normalen“ Laborabfall. Dieses Be-enthaltende Gefäß kann dann selbst im herkömmlichen Laborabfall als giftiger Feststoff entsorgt werden. Spezielle Entsorgungsvorschriften für Be und seine Verbindungen sind uns für kein einziges Bundesland bekannt, der örtlich zuständige Entsorgungsbeauftragte erteilt hierzu nähere Auskünfte. Auf diesem Wege ist Vermischung der Abfälle, und somit die Kontamination von Mitarbeitern, ausgeschlossen. Unserer Erfahrung nach ist dies die beste Möglichkeit, die Kommilitonen im Labor zu schützen. Die Entsorgung von Be-Verbindungen erfolgt vorzugsweise in Lösung, so kann nichts stauben. Im Allgemeinen verwenden wir (eis)-kaltes Wasser zur Entsorgung von Halogeniden (stark exotherme Reaktion) und übliche organische Lösungsmittel für metallorganische oder koordinationschemische Verbindungen des Berylliums. Um dabei die Freisetzung von Be-haltigen Aerosolen zu unterbinden, setzen wir größere Schlenkgefäße (vom zehnfachen Volumen bezogen auf die zu erhaltende Abfalllösung) unter externer Kühlung ein. Wird trotzdem ein Aerosol freigesetzt, so kann dieses schnell und einfach mit Haarspray oder Kleberspray gebunden und aufgewischt werden. Extrem reaktive Verbindungen wie z.B. Be-Alkyle oder Be-Hydride, werden vorsichtig und sanft durch eine Lösung von $5 \%{ }^{t} \mathrm{BuOH}$ in Toluol unter Schutzgas zersetzt. Man denke daran, dass sich hierbei Wasserstoff entwickelt und man hat daher wieder die Freisetzung von Aerosolen zu vermeiden. Die erhaltenen Lösungen können als giftiger Flüssigabfall entsorgt werden.

Die meisten Chemiker verwenden zur Reinigung der Glasgeräte ein Bad, das eine Lösung von $\mathrm{KOH}$ in Isopropanol enthält. Als zusätzliche Schutzmaßnahme empfehlen wir die Verwendung eines eigenen Bads für Glasgerätschaften, die mit $\mathrm{Be}$ und seinen Verbindungen im Kontakt waren.

Arbeiten mit dem Handschuhkasten: Wir empfehlen zusätzliche Handschuhe (Größe L oder XL aus Latex oder Nitril) über den Handschuhen im Handschuhkasten zu tragen, um die Kontamination der Handschuhe des Kastens selbst auszuschließen. Zudem ist so ein Paar zusätzlicher Handschuhe für die Verhinderung von Kontamination sehr nützlich, da diese umstülpend ausgezogen und entsorgt werden können. Die Vermischung von Glasgeräten anderer Mitarbeiter muss natürlich ausgeschlossen werden, hierzu verwenden wir farbige Kabelbinder zur Markierung an den Glasgeräten. Zur Abfallentsorgung im Handschuhkasten setzen wir ein darin befindliches Gefäß ein, welches schließlich in einer bruchsicheren und dicht schließenden Umverpackung entsorgt wird. Staub kann sich durch elektrostatische Aufladung auch an den Außenseiten von Kunststoffgefäßen oder Glasgeräten anlagern. Daher empfehlen wir das feuchte Abwischen, oder das Untertauchen von Gerätschaften in Wasser, unmittelbar nach dem Ausschleußen aus dem Handschuhkasten. Während aller Arbeiten am Handschuhkasten tragen wir FFP3-Partikelfiltermasken oder ABEK-P3-Halbmasken.

Neben der schon beschriebenen Schutzmaßnahmen stellen wir auch unsere Erfahrungen des arbeitstäglichen Umgangs mit Be und seinen Verbindungen vor.

Handhabung von Be-Verbindungen: Viele Chemiker arbeiten ohnehin mit luft- und/oder feuchtigkeitsempfindlichen Stoffen und sind daher gewöhnt unter Schlenkbedingungen zu arbeiten. Für die erfolgreiche Synthese von bisher unbekannten Verbindungen des Berylliums sollte man aber im Hinterkopf behalten, dass diese häufig deutlich reaktiver und empfindlicher gegenüber Luftbestandteilen sind als verwandte Verbindungen, zum Beispiel denen des Magnesiums oder des Aluminiums. Jeder präparativ arbeitende Chemiker hat sicher seinen eigenen persönlichen Stil der sogenannten „StandardSchlenktechnik“. Dagegen ist zunächst nichts zu sagen, sofern man diesen Stil selbst stets kritisch prüft. Aufgrund unserer Erfahrungen der letzten Jahre können wir noch einige weitere Empfehlungen geben, denn so manche Be- 
Verbindung hat doch eine ganz eigene Behandlung erfordert.

Zuvorderst muss man sich sicher sein, dass man trockene Lösungsmittel einsetzt - das heißt, der per KarlFischer-Titration bestimmte Restwassergehalt liegt unter $0,1 \mathrm{ppm}$. Be-Verbindungen sind meist starke Elektrophile, Lewis-sauer und chemisch sehr hart nach dem PearsonKonzept. Die Wahl des Lösungsmittels hängt natürlich von der angedachten Reaktion ab. Soweit wir es bisher beurteilen können, sind metallorganische und koordinationschemische Verbindungen des $\mathrm{Be}$ in vielen Lösungsmitteln zumindest metastabil, zum Beispiel in Kohlenwasserstoffen (Heptan, Hexan, Pentan, etc.), in Aromaten (Benzol, Toluol), in koordinierenden Lösungsmitteln (Pyridin, Thf, Et ${ }_{2} \mathrm{O}$, Mtbe, Acetonitril, Benzonitril) oder in polaren Lösungsmitteln $\left(\mathrm{CH}_{2} \mathrm{Cl}_{2}, 1,2-\right.$ Difluorobenzol, Fluorobenzol, „Benzoltrifluorid") und selbstverständlich in flüssigem Ammoniak. Abhängig vom Koordinationsmodus und der Koordinationszahl am Be-Kation sind die Verbindungen in Lösungen häufig stabil. Als Faustregel kann man annehmen, dass je kleiner die Koordinationszahl und je chemisch weicher die Liganden, desto instabiler wird die Verbindung in Lösung sein. Als typische Nebenreaktionen mit dem Lösungsmittel haben wir die Abspaltung von Halogeniden oder die Etherspaltung beobachtet. Im Falle von koordinierenden Lösungsmitteln kann es sehr problematisch bis unmöglich werden das Lösungsmittel wieder vom Be-Kation zu entfernen.

Berylliumverbindungen sind gegenüber manchen Schlifffetten empfindlich, meistens gegenüber solchen auf Polysiloxan-Basis. Wir empfehlen daher die Verwendung von Stopfen auf J-Young-Basis (fettfrei) oder wenigstens den Einsatz von Schliffkappen (so dass das Fett außen und nicht innen ist), um jede Verunreinigung durch Fett $\mathrm{zu}$ vermeiden. PTFE-basierende Fette sind die Fette der Wahl. Im Anhang findet sich eine Auswahl der von uns eingesetzten Gerätschaften und Techniken. Wir betonen hier nochmals, dass der talentierte Chemiker immer die Schlenk-Technik vor der Verwendung eines Handschuhkastens bevorzugt, da die Atmosphäre des Handschuhkastens auch andere Stoffe enthält, mit denen sehr reaktive Verbindungen dann auch reagieren werden. Provokativ ausgedrückt enthält ein Handschuhkasten unter anderem auch Schutzgas (und jeden anderen Stoff mit Dampfdruck). Wasser ist mit seinem Gleichgewichtspartialdruck über dem verbauten Trockenmittel vorhanden - eine hygroskopischere Verbindung als das Trockenmittel selbst wird also zur Einstellung eines neuen Gleichgewichts führen und so die Oberflächen des Handschuhkastens und das Trockenmittel trocknen. In Handschuhkästen sollten daher auch keine Kohlenhydrate aufbewahrt werden, also keine Papierwischtücher, Papieretiketten, Bleistifte. Ist eine Verbindung nur hygroskopisch genug, so wird sie solchen Stoffen das Wasser entreißen. Das Vortrocknen von Papierwischtüchern in einem Trockenschrank hilft dabei selbstverständlich kaum.

Vor dem Beginn der Umsetzung muss man natürlich adsorbierte Feuchtigkeit auf den Oberflächen der
Reaktionsgefäße weitestgehend ausschließen. Wir bewahren dazu unsere Gefäße immer mindestens vier Stunden in einem Trockenschrank auf, der bei $130-160$ ${ }^{\circ} \mathrm{C}$ betrieben wird. ${ }^{[51]}$ Dann schließen wir die noch heißen Gefäße an die Schutzgasanlage an und heizen sie mit einem Bunsen- oder Teclubrenner stellenweise so lange unter Vakuum aus, bis man die gelbe Natriumflamme des Glases beobachtet. Dann lässt man am dynamischen Vakuum abkühlen, gibt Schutzgas hinzu und wiederholt die gesamte Prozedur des Sekurierens; wir empfehlen mindestens noch zwei weitere Male. Man denke daran, dass ein Heißluftgebläse (die „Heat Gun“) lediglich Temperaturen bis zu $650{ }^{\circ} \mathrm{C}$ erreicht, das Glas ist deutlich kälter. Solche Temperaturen reichen bei wirklich hygroskopischen Verbindungen oft nicht aus, um „wasserfrei“ $\mathrm{zu}$ arbeiten. Ob es so trocken genug wird, hängt nur von der Reaktivität der eingesetzten Verbindungen $\mathrm{ab}$. Mit einem Bunsen- oder Teclubrenner erhält man hingegen praktisch den kleinstmöglichen Restfeuchtigkeitsgehalt in Glasgefäßen (die letzte Schicht Wasser kann von einer Glasoberfläche nur bei $400{ }^{\circ} \mathrm{C}$ im Ultrahochvakuum entfernt werden). In so ausgeheizten Glasgeräten können unserer Erfahrung nach auch dünne Filme elementaren Caesiums auf der Glasoberfläche mehr als sieben Tage lang unverändert aufbewahrt werden, falls das verwendete Schutzgas sauber genug ist. Sehr gut zur Entfernung von Restfeuchtigkeit ist auch das Ausbacken von mit gasförmigem Phosgen (wenige hundert mbar absolut) befüllten Glasgefäßen

Manchmal müssen Reaktionsgefäße in besonderer Weise für Kristallisationszwecke vorbereitet werden. Um die Glasoberfläche zu hydrophobieren geben wir eine kleine Menge (ca. $1 \mathrm{~mL}$ ) von $\mathrm{HN}\left(\mathrm{SiMe}_{3}\right)_{2}$ in die Gefäße, legen einen leichten Unterdruck an und schließen den Hahn. Dann wärmt man die Flüssigkeit mit einem Heißluftgebläse auf bis sie bis zum Hals des geschlossenen Gefäßes refluxiert. Dann öffnet man den Hahn zum Vakuum und destilliert das übrige $\mathrm{HN}\left(\mathrm{SiMe}_{3}\right)_{2}$ in die Kühlfalle ab. Danach beginnen wir mit oben beschriebener Prozedur.

Es muss angemerkt werden, dass Berylliumhalogenide mit Borosilicatglas 3.3 (Duran, Pyrex) reagieren und sogar Kieselglas („Quarzglas“) wird bei höheren Temperaturen und Drücken bei oder oberhalb von 1 bar Druck, den typischen Synthesebedingungen der BeHalogenide $\mathrm{BeX}_{2}(\mathrm{X}=\mathrm{Cl}, \mathrm{Br}, \mathrm{I})$, angegriffen. ${ }^{[52]}$ Dagegen ist "Quarzglas“ nach unseren Erfahrungen wesentlich beständiger. Borosilicatglas kann gut zur Sublimation dieser Verbindungen unter Hochvakuum eingesetzt werden und man beobachtet keine Reaktion.

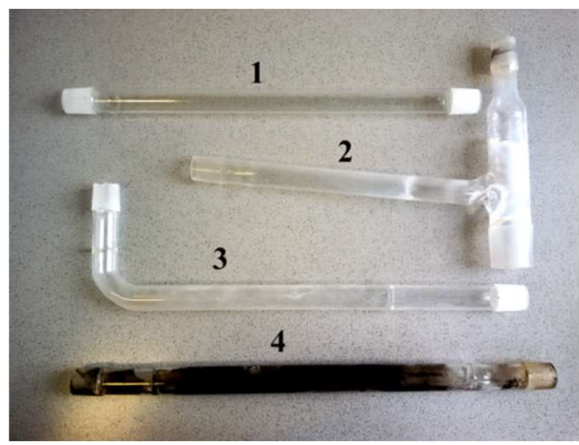


Abbildung 3. Verschiedene, zur Synthese von Be-Halogeniden bei höheren Temperaturen eingesetzte Glasrohre / 1- neu / 2 $\mathrm{BeCl}_{2} /-3-\mathrm{BeF}_{2}$ durch Zersetzung von $\left(\mathrm{NH}_{4}\right)_{2} \mathrm{BeF}_{4} / 4-\mathrm{BeCl}_{2}$ $\left(\mathrm{BeO}+\mathrm{C}+\mathrm{Cl}_{2} \rightarrow \mathrm{BeCl}_{2}+\mathrm{CO}\right)$. Bild: D. Naglav

\section{Was ist bei einem Notfall zu tun?}

Je mehr man über Unfälle und unliebsame Situationen im Vorfeld nachgedacht hat, desto geringer werden deren Auswirkungen sein, da man ruhiger und vorbereiteter ist. Nota bene: Man schreibe diese Gedanken nieder! Sicherheitsbeauftragte, Amtspersonen der Unfallkassen und im schlimmsten Falle sogar Staatsanwälte können solche Gedanken einfordern, so dass man sie lieber gleich in Form einer "Betriebsanweisung und Gefährdungsbeurteilung” festgehalten hat.

Passiert etwas Unerwartetes, wie das Entstehen eines Lecks in der geschlossenen Apparatur, ein Bruch von Glasgeräten oder etwas anderem, und man selbst oder der Abzug wird mit Be-haltigen Stäuben, Aerosolen oder Flüssigkeiten kontaminiert, so kann man mit nahezu an Sicherheit grenzender Wahrscheinlichkeit davon ausgehen, dass einen oben beschriebene Schutzmaßnahmen auch wirklich schützen. Die Mitarbeiter sind umgehend zu verständigen, so dass jeder nicht genehmigte Zugang zum Labor, vor allem von nicht ausreichend geschützten Personen, verhindert wird. Ist das Digestorium kontaminiert, so kann Staub mit feuchten Tüchern und Wasser aufgenommen und gebunden werden. Hierbei hydrolysiert die ausgetretene Verbindung und wandelt sie in hydroxidische Spezies um, welche durch feuchtes Abwischen entfernt werden. BeO, welches sich bei der Verbrennung von metallorganischen Verbindungen des Berylliums an Luft bilden könnte, sollte genauso aufgenommen werden. Die Feuchtigkeit verhindert, dass der Staub aufgewirbelt wird. Der hierbei erzeugte Abfall soll wie oben beschrieben entsorgt werden. Nach weiterer Inspektion auf bislang übersehene Kontamination empfehlen wir das gesamte Digestorium und seinen Inhalt zu reinigen, wie man es routinemäßig ohnehin öfter macht. Hat man eine pulverförmige, leicht staubende Verbindung verschüttet, so kann man diese mit einem Wassernebel besprühen. Man sprühe jedoch nicht direkt auf das Pulver, sondern in die Luft darüber, um es nicht wegzublasen. Auch Haarspray eignet sich hierfür wieder besonders gut, wbei man wiederum darauf achte, nicht direkt auf das Pulver zu sprühen, damit das Aerosol sich langsam auf das verschüttete Pulver legen kann. Danach kann alles ohne Staubentwicklung aufgenommen werden. In einem laufenden Abzug haben die beiden Methoden nur eine begrenzte Anwendbarkeit, außerhalb eines Abzugs sind sie hingegen unschlagbar. Wir empfehlen diese Technik im Übrigen auch für verschüttete Pulver von Nanomaterialien und von radioaktiven Verbindungen.
Findet eine Kontamination oder gar eine Inkorporation statt, ohne dass man die oben beschriebene persönliche Schutzausrüstung getragen hat, so ist man selbst Schuld (aber man selbst, sowie auch der Arbeitgeber sind für die Schäden verantwortlich)! In diesem Fall kann man nur noch die üblichen Notfallmaßnahmen durchführen (Notdusche, Augendusche, Haut mit reichlich fließendem Wasser abwaschen, den Arzt aufsuchen, oder den Notarzt rufen) - aber das Kind ist bereits in den Brunnen gefallen.

\section{Wie analysiert man seine Verbindungen?}

Die Charakterisierung von Be-Verbindungen wird im Allgemeinen genauso durchgeführt wie bei allen anderen chemischen Verbindungen. Typische Methoden sind die Röntgenbeugung am Einkristall und am Pulver, die Elementaranalyse, die NMR-, IR-, Raman- und UV/VISSpektroskopie, und so weiter. ${ }^{[53-58]}$ Führt man Röntgenbeugungsexperimente an Be-Verbindungen durch, so sollte man daran denken, dass die Verbindungen nur Leichtatome enthalten können, so dass man dann auf eine Absorptionskorrektur verzichten kann. Beryllium ist ein sehr nützlicher Kern für fortgeschrittene NMRspektroskopische Untersuchungen: ${ }^{9} \mathrm{Be}$ ist ein Quadrupolkern mit einem Kernspin von 3/2, einer natürlichen Häufigkeit von $100 \%$, und zudem ein sehr empfindlicher Kern für die NMR-Spektroskopie. Daher kann man in kurzer Zeit sehr fein aufgelöste ${ }^{9}$ Be-NMRSpektren aufnehmen, die eine in situ Beobachtung von Be-Verbindungen erlauben. Die breite der Signale hängt von der Symmetrie und der Koordinationszahl der untersuchten Spezies ab. Der Bereich der chemischen Verschiebung liegt typischerweise zwischen -20 bis +30 ppm. Als Referenz dient eine 0.43 molare Lösung von $\mathrm{BeSO}_{4} \cdot 4 \mathrm{H}_{2} \mathrm{O}$ in $\mathrm{D}_{2} \mathrm{O}(0 \mathrm{ppm}){ }^{[58]}$ Weitere Details finden sich in der angegebenen Literatur.

\section{Wie charakterisiert man seine Be-haltigen Materialien?}

Die wahrscheinlich beste Methode für die Analyse von (zumindest teilweise) kristallinen Materialien ist die Röntgenbeugung. ${ }^{[59]}$ Mit ihrer Hilfe lassen sich kristalline Phasen identifizieren, die Gitterparameter können bestimmt und an dünnen Filmen können Stressanalysen durchgeführt werden. Berylliummetall kristallisiert bei Standardbedingungen im hexagonalen Raumgruppentyp $\mathrm{P6}_{3} / \mathrm{mmm}$ (Mg-Typ), was $\mathrm{zu}$ einem einfachen Diffraktogramm führt das eindeutig von anderen kristallinen Be-Verbindungen wie die der Oxide, Nitride und Carbide unterschieden werden kann. Die Analyse von Be-Filmen wird durch die Ausbildung dünner Oxidschichten bei Luftkontakt erschwert. Ausgewählte ex-situ Analysenmethoden können für die Tiefenprofilierung herangezogen werden. Auch wenn die energiedispersive Röntgenspektroskopie (EDX) eine Standardmethode ist, ist sie oft für die Detektion von Be ungeeignet, was aber vom Gerät abhängt. ${ }^{[61]}$ Die meisten EDX-Spektrometer können Elemente mit $\mathrm{Z}<6$ (Kohlenstoff) nicht mehr nachweisen, da die Erzeugung von charakteristischer Röntgenstrahlung mit kleinerem $Z$ abnimmt: Die Energie der emittierten Röntgenstrahlung 
ist oft so gering, dass deren Absorption durch die Probe und durch den Detektor zur starken Abnahme der Signalintensität führt. Nach unserer Erfahrung eignet sich die EDX-Spektroskopie für die Reinheitsanalyse von BeMetall, da dieses durch den Produktionsprozess bedingt oft mit Ca und Halogeniden verunreinigt ist. Die AugerSpektroskopie ${ }^{[60]}$ ist sehr empfindlich auf Leichtelemente, da der KVV-Übergang von metallischem Beryllium bei $105 \mathrm{eV}$ beobachtet werden kann. Die Röntgenphotoelektronen-Spektroskopie ${ }^{[60]}$ stellt eine oberflächenempfindliche Methode zur Bestimmung der chemischen Umgebung eines Elements dar; der 1s Peak von metallischem Be bei 110,5 eV kann von dem 1s-Peak des an Sauerstoffatome gebundenen Be-Atoms bei 113 $\mathrm{eV}$, des an $\mathrm{N}$-Atome gebundenen bei $114 \mathrm{eV}$ und des an C-Atome gebundenen bei $112 \mathrm{eV}$ unterschieden werden. Die Sekundärionenmassenspektroskopie (SIMS) stellt eine sehr empfindliche Methode für die Analyse von BeVerbindungen in dünnen Filmen dar. Beryllium ist leicht zu ionisieren, so dass dessen Nachweisgrenze im subppm-Bereich liegt, so dass sogar Be-Diffusionsstudien möglich werden. Die Atomemissionsspektroskopie (AES) ist die Standardmethode zur Quantifizierung von Beryllium. Zum Beispiel kann man auch die Laborluft durch geeignete Filter saugen und diese dann auf Be untersuchen. So lässt sich eine Atemluftüberwachung selbst einrichten.

\section{Welche Besonderheiten haben wir beim Arbeiten mit Beryllium beobachtet?}

Metallorganische Verbindungen des Berylliums sind hochreaktiv: Während unserer Untersuchungen am Beryllium hat es mehrere Situationen gegeben, in denen die extreme Elektrophilie des Berylliums offenkundig wurde. $^{[62]}$ Bei dem Versuch, die Verbindung $\mathrm{Cp} * \mathrm{BeF}$ $\left(\mathrm{Cp}^{*}=1,2,3,4,5\right.$-methylcyclopentadienyl $)$ duch oxidative

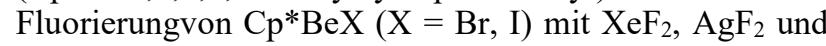
$p-\mathrm{F}-\mathrm{C}_{6} \mathrm{H}_{4} \mathrm{IF}_{2}$ herzustellen, wollten wir die beiden Feststoffe im Handschuhkasten unter Argonatmosphäre zusammengeben. Die Reaktion verlief jedoch nicht wie geplant. Nachdem beide Verbindungen separat eingewogen worden waren, legten wir die Verbindungen nebeneinander, ohne sie $\mathrm{zu}$ vermengen. Zwischen den beiden Feststoffen begann es fürchterlich mit greller Flamme zu brennen, ein schwarz-violetter Rauch stieg auf und nur glühende Asche blieb zurück. Die Triebkraft der Reaktion war vermutlich die exotherme Bildung von $\mathrm{HF}$ und $\mathrm{BeF}_{2}$. Einerseits zeigt dies die Reaktionsfreudigkeit der oxidativen Fluorierung, andererseits unterstreicht es die Reaktivität metallorganischer Verbindungen des Berylliums, da wir bei vergleichbaren Umsetzungen mit anderen $\mathrm{Cp}^{*} \mathrm{MX}_{\mathrm{n}}-$ Verbindungen $(\mathrm{M}=$ Metall, $\mathrm{X}=$ Halogen) nie ein solches Verhalten beobachtet haben. Da $\mathrm{BeEt}_{2}$ und $\mathrm{Be}^{i} \mathrm{Bu}_{2}$ bei Zimmertemperatur flüssig sind, haben wir mit diesen Stoffen mit Hilfe der Spritzentechnik gearbeitet. Nach der Zugabe der Verbindungen zu Reaktionslösungen wollten wir die Spritzen unter dem Abzug entsorgen und zogen den Stempel der vermeintlich leeren Spritze heraus. Die verbliebene Restmenge führte $\mathrm{zu}$ einer großen Flamme und feiner, weißer Staub bildete sich. Im Vergleich zu den wohlbekannten, analogen Verbindungen des Aluminiums oder Zinks erscheinen die Berylliumverbindungen in unseren Händen reaktiver. Aus diesem Grund entwickelten wir die oben beschriebene Vorgehensweise zur Entsorgung.

Berylliumverbindungen sind süß, ja tatsächlich: Die meisten Chemiker wissen, dass das Beryllium für die Süße mancher seiner Verbindungen berühmt ist, was auch dazu führte, dass das Element in Frankreich als Glucinium bezeichnet wurde (vor Einführung der IUPACNomenklatur im Jahr 1957). Diese Beobachtungen rühren von Geschmacksexperimenten her, welche ja in früheren Jahrhunderten nicht unüblich waren. ${ }^{[1]}$ Durch unsere intensiven Untersuchungen an $\mathrm{Cp}^{*}$-Komplexen des Berylliums können wir Erfahrungen nennen, die die früheren Befunde stützen. Aufgrund der hohen Flüchtigkeit der $\mathrm{Cp}^{*}$-Komplexe fiel uns auf, dass Verbindungen wie $\mathrm{Cp} * \mathrm{BeR}\left(\mathrm{R}=\mathrm{Cl}, \mathrm{Br}, \mathrm{I}, \mathrm{Cp}^{*}\right)$ einen sehr intensiven, aber zugleich auch fantastischen Geruch aufweisen, vergleichbar mit dem eines starken Parfüms. Solch ein parfümartiger süßer Duft wurde unseres Wissens noch nicht berichtet und unterstreicht die Schönheit des Elements. Wie alles im Leben hat die Medaille aber zwei Seiten - auf die dunklere sind wir in diesem Bericht schon eingegangen. Wir machten diese Beobachtungen, als wir bei Zimmertemperatur Einkristalle für die Röntgenbeugung auswählten. Die Zahl der Kristalle, mit einer Größe von etwa 0.4 × 0.22 × 0.1 $\mathrm{mm}^{3}$, lag nur zwischen vier bis zehn, zudem waren die Kristalle mit perfluoriertem Öl bedeckt. Am Mikroskop fiel uns bei der Fixierung der Kristalle auf kleine Nylonschlaufen stets der starke Geruch auf, obwohl die Menge der Kristalle und damit wohl auch die Konzentration in der Luft überaus gering war. Präparierten wir die Kristalle im kalten Öl bei Temperaturen zwischen -50 und $-40{ }^{\circ} \mathrm{C}$, so nahmen wir keinen Geruch wahr, was vermutlich an dem deutlich kleineren Sublimationsdruck bei diesen Temperaturen liegt. Weiterhin fiel uns ein süßer Geruch auf, als eine kleine Menge an $\mathrm{BeCl}_{2}$ aus einer Apparatur wegen $\mathrm{zu}$ großen Überdrucks hinausgeblasen wurde.

Cave! Da Filtermasken von Partikeln, die kleiner als 0,6 $\mu \mathrm{m}$ sind, durchdrungen werden, sollten für solche Fälle Halbmasken mit Aktivkohlefilter (z.B. oben erwähnte ABEK-P3-Filter) eingesetzt werden. Dann wird man jedoch die Verbindungen nicht mehr riechen - riecht man trotzdem etwas, so weiß man dass es an der Zeit ist den Filter zu wechseln (außerhalb des Gefahrenbereichs).

\section{Wie ist der gegenwärtige Stand der Berylliumchemie?}

Die folgenden Absätze erheben keinen Anspruch auf Vollständigkeit sondern stellen eine Auswahl an Arbeiten dar, die wir als besonders lesenswert erachten.

\section{Koordinationsverbindungen}

Die wässrige Koordinationschemie des $\mathrm{Be}$ ist viel besser untersucht als die in organischen Lösungsmitteln, so dass über viele Verbindungen bereits in 
hervorragenden Übersichtsartikeln diskutiert wurde. Die Chemie des Berylliums wird von seiner Gier nach Elektronen(paaren) dominiert. In wässriger Lösung zeigen seine Komplexe tetraedrische Koordination, welche für alle Be-Verbindungen die bevorzugte ist. Das Be zeigt auch eine Tendenz zur Bildung sechsgliedriger Ringe in denen drei $\mathrm{Be}^{2+}$-Kationen über ein Anion verbrückt sind $\left[\mathrm{Be}_{3}\left(\mu-\mathrm{A}^{-}\right)_{3}(\text { Donor })_{6}\right]^{3+}$, und jedes Be-Atom tetraedrisch koordiniert wird.

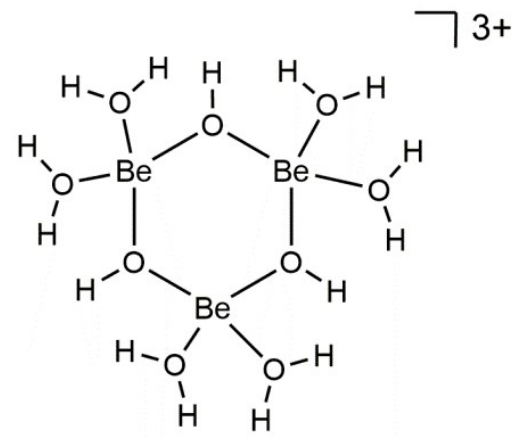

Abbildung 4. $\left[\mathrm{Be}_{3}\left(\mu-\mathrm{OH}^{-}\right)_{3}\left(\mathrm{H}_{2} \mathrm{O}\right)_{6}\right]^{3+}$-Kation

\section{„Recent Contributions to the Aqueous Coordination Chemistry of Beryllium" - H. Schmidbaur, Coord. Chem. Rev. 2001, 215, 223-242. ${ }^{[3]}$}

Koordinationsverbindungen in nicht-wässrigen Systemen sind praktisch nur in klassisch organischen Medien wie Toluol, Tetrahydrofuran, $\mathrm{CH}_{2} \mathrm{Cl}_{2}$ und so weiter, untersucht worden. Eine der wenigen Ausnahmen stammt aus der Feder eines der Autoren über die Koordinationschemie des Berylliums in wasserfreiem Ammoniak, welche zu Ergebnissen wie dem $\mathrm{Be}^{2+}$-Kation als Fluoridionenakzeptor oder zum $\left[\mathrm{Be}\left(\mathrm{NH}_{3}\right)_{4}\right]^{2+}$-Kation geführt haben. Durch gezielte Hydrolyse in diesem Medium konnte auch das sechsgliedrige Ringkation $\left[\mathrm{Be}_{3}(\mu-\mathrm{OH})_{3}\left(\mathrm{NH}_{3}\right)_{6}\right]^{3+}$ beobachtet werden. ${ }^{[63,64]}$

„Reactions of Beryllium Halides in Liquid Ammonia: The Tetraammine Cation $\left[\mathrm{Be}\left(\mathrm{NH}_{3}\right)_{4}\right]^{2+}$, its Hydrolysis Products, and the action of $\mathrm{Be}^{2+}$ as a Fluoride-Ion Acceptor" - F. Kraus, S. A. Baerl, M. R. Buchner, A. J. Karttunen, Chem. Eur. Jour. 2012,

$$
18(7), 2131-2142 .^{[63]}
$$

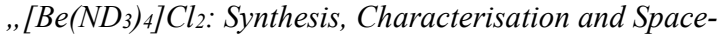
Group Determination Guided by Solid-State Quantum Chemical Calculations" - F. Kraus, S. A. Baerl, M. Hoelzl, A. J.

Karttunen, Eur. J. Inorg. Chem. 2013, 24, 4184-4190. [64]

Das Grundwissen der Koordinationschemie des Berylliums wurde von zwei Pionieren zusammengefasst, nämlich von Prof. Kurt Dehnicke $(\dagger 2011$, sein Tod stand nicht mit Be im Zusammenhang) und Prof. Bernhard Neumüller. Die exzellente Zusammenschau zeigt alle Fortschritte der Be-Chemie bis zum Jahr 2008 und beschreibt das komplizierte chemische Verhalten der Berylliumhalogenide mit zahlreichen Liganden in nichtwässrigen Systemen.
,Research Report - Recent Advances in the Chemistry of Beryllium" - K. Dehnicke, B. Neumüller, Z. Anorg. Allg. Chem. 2008, 634, 2703-2728. ${ }^{[65]}$

In den letzten Jahrzehnten sind neben diesem Bericht nur wenige Manuskripte über Be-Komplexe erschienen. ${ }^{[66-90]}$ Viele Untersuchungen konzentrierten sich auf die Synthese von NHC-Komplexen (Nheterocyclische Carbene), welche zur Entdeckung der Aktivierung dieser Carbene in beryllium-induzierten Ringerweiterungsreaktionen und zur Synthese von einem $\mathrm{NHC}-\mathrm{BePh}_{2}$-Komplex geführt haben. ${ }^{[82,83]}$ Es gelang auch das erste Beispiel eines Berylliumborhydrids, als $\mathrm{NHC}: \mathrm{Be}\left(\mathrm{BH}_{4}\right)_{2}$, strukturell zu charakterisieren; über die Verbindung wurde fortwährend, seit ihrem ersten Bericht vor über 70 Jahren, diskutiert. ${ }^{[85]}$ Einer der führenden Wissenschaftler auf diesem Gebiet ist Prof. M. Hill, der auch die Reduktionschemie und das Reaktionsverhalten von $\beta$-Diketiminatberylliumkomplexen (N-acetyl-Nacetonat $=$ nacnac) untersuchte. In seinen Arbeiten konnte er zeigen, dass koordinierte Berylliumalkylkomplexe sowohl die Etherspaltung von thf auslösen als auch Polymerisationsreaktionen einleiten können. ${ }^{[1,82,85,86]}$ Die Autoren des vorliegenden Artikels haben sich der Synthese von heteroleptischen Scorpionat-Komplexen des $\mathrm{Be}$ angenommen (Trispyrazolylborat (Tp)) und Untersuchungen über die Natur von homoleptischen, linearen Be-amiden, wie $\mathrm{Be}\left(\mathrm{N}\left(\mathrm{SiMe}_{3}\right)_{2}\right.$, durchgeführt. ${ }^{[88 \mathrm{a})}$ b),89]
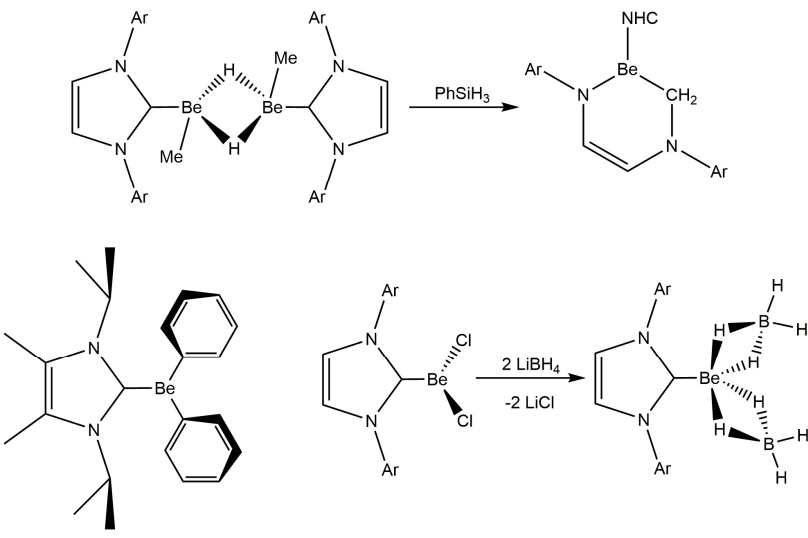

Abbildung 5. Berylliumchemie mit $N$-heterocyclischen Carbenen (NHC)

„Modern Organometallic and Coordination Chemistry of Beryllium" - K. J. Iversen, S. A. Couchman, D. J. D. Wilson, J. L. Dutton, Coord. Chem. Rev. 2015, 297-298, 40-48. [66]

\section{Metallorganische Verbindungen}

In den letzten 15 Jahren scheint die metallorganische Chemie des Berylliums aus ihrem Dornröschenschlaf erwacht zu sein, da mehrere Gruppen mit Untersuchungen $\mathrm{zu}$ solch reaktiven Verbindungen begonnen haben. ${ }^{[90-100]}$ Wichtige Beiträge lieferten die Arbeiten von Prof. P. 
Power, der über die Darstellung von homoleptischem $\mathrm{BeMes}_{2} \cdot\left(\mathrm{Et}_{2} \mathrm{O}\right)(\mathrm{Mes}=1,3,5$-trimethylbenzol $)$ und von heteroleptischen, sterisch überfrachteten Verbindungen, wie $\operatorname{ArBeX} \cdot\left(\mathrm{Et}_{2} \mathrm{O}\right)\left(\mathrm{Ar}=\mathrm{C}_{6} \mathrm{H}_{3}-2,6-\mathrm{Mes}_{2} / \mathrm{X}=\mathrm{Cl}, \mathrm{Br}\right.$, $\left.\mathrm{SMes}, \mathrm{NHPh}, \mathrm{NHSiPh}_{3}, \mathrm{NSiMe}_{3}\right)$, gepaart mit detaillierten ${ }^{9}$ Be-NMR-spektroskopischen Untersuchungen, berichtete. ${ }^{[97,98]}$ Prof. E. Carmona veröffentlichte die strukturelle Charakterisierung von Beryllocen BeCp*2 $\left(\mathrm{Cp}^{*}=\right.$ pentamethylcyclopentadienyl $)$ und mehreren seiner Derivate. ${ }^{[100]}$
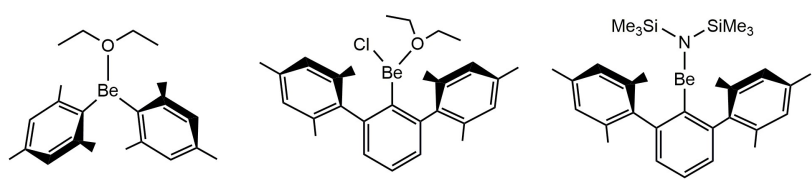

Abbildung 6. Be-Chemie mit sterisch anspruchsvollen organischen Liganden

„Recent Developments in the Chemistry of Beryllocenes” - R. Fernández, E. Carmona, Eur. J. Inorg. Chem. 2005, 3197$3206 .{ }^{[100]}$

„Annual Report on Alkaline and Alkaline Earth Metals” - M. S. Hill, Inorg. Chem. 2013, 109, 18-27. ${ }^{[81]}$

\section{Theoretische Untersuchungen}

In mehreren Übersichtsartikeln sprechen die Autoren vom Mangel an experimentellen Untersuchungen zur BeChemie im Vergleich mit der Vielzahl an theoretischen Arbeiten auf diesem Gebiet. ${ }^{[3]}$ Letzteres liegt vermutlich an der Einzigartigkeit des Be aufgrund seiner exponierten Stellung im Periodensystem, welche zu ungewöhnlichen Bindungseigenschaften führt. Das Interesse an den Eigenschaften der Be-Verbindungen sollte für Experimentatoren ein Anreiz sein, um Theoretikern zu zeigen, ob sie richtig oder falsch liegen. Die meisten Arbeiten handeln von den besonderen Bindungseigenschaften von $\mathrm{Be}$ aufgrund seiner beiden leeren p-Orbitale und der resultierenden hohen LewisAcidität. ${ }^{[101-108]}$ Kürzlich hat man auch versucht herauszufinden, welche Eigenschaften Liganden haben müssen, damit sie sich zur Synthese von Be(I)Verbindungen eignen. ${ }^{[106]}$ Ein weiteres Forschungsgebiet sind ungewöhnliche Bindungssituationen wie beispielsweise bei der Bindung von $\mathrm{Be}$ an Edelgase, ${ }^{[109,110]}$, die Bindung von Be-Clustern ${ }^{[111,112]}$ und Bindungen in Koordinationsverbindungen. ${ }^{[113,114]}$
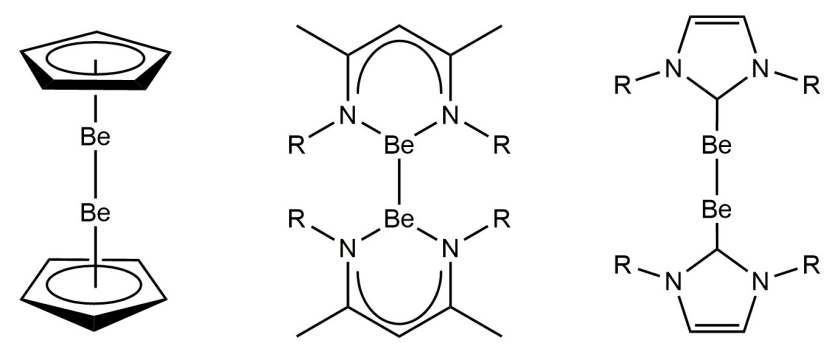

Abbildung 7. Theoretische Be-Chemie

„Multimetallocenes. A Theoretical Study” - A. Velazquez, I. Fernández, G. Frenking, G. Merino, Organometallics 2007, 26, $4731-4736 .^{[102]}$

„Beryllium chemistry the safe way: a theoretical evaluation of low oxidation state beryllium compounds" - S. A. Couchman, N. Holzmann, G. Frenking, D. J. D. Wilson, J. L. Dutton, Dalton Trans 2013, 42, 11375-11384. ${ }^{[106]}$

„Comparison of the Mechanism of Borane, Silane, and Beryllium Hydride Ring Insertion into N-Heterocyclic Carbene $C-N$ Bonds: A Computational Study" - K. J. Iversen, D. J. D.

Wilson, J. L. Dutton, Organometallics 2013, 32 (21), 62096217.[107]

„On the stability of noble gas bound 1-tris(pyrazolyl)-borate beryllium and magnesium complexes" - S. Pan, R. Saha, P. K.

Chattaraj, New J. Chem. 2015, 39, 6778-6786. ${ }^{[109]}$

"Creating $\sigma$-Holes through the Formation of Beryllium Bonds" - O. Brea, O. Mó, M. Yáñez, I. Alkorta, J. Elguero, Chem. Eur. J. 2015. 21, 12676-12682. ${ }^{[113]}$

\section{Materialchemie}

Aus der Sicht des Materialchemikers ist das Beryllium aufgrund seiner einzigartigen Eigenschaften ein wahrer Traum. Eine geringe Dichte, vergleichbar mit der des $\mathrm{Mg}$ aber kleiner als die des Al (den beiden ,klassischen“ Leichtmetallen), in Verbindung mit einem hohen Schmelzpunkt und großem Elastizitätsmodul machen es für die Anwendung in Luft-und Raumfahrt attraktiv. Be und sein Oxidationsprodukt $\mathrm{BeO}$ gelten als „ausgesprochen giftig“, insbesondere als Staub, aber auch Hautkontakt wird beim industriellen Umgang als problematisch eingestuft, so dass der Der Traum zum Albtraum mutiert. Aufgrund der enormen Sicherheitsvorkehrungen, die für die industrielle Verarbeitung von Be nötig sind, wird es nur dort eingesetzt, wo Kosten typischerweise keine Rolle spielen. Beryllium ist praktisch transparent für Röntgenstrahlen, weist nur einen kleinen Einfangsquerschnitt für thermische Neutronen, aber einen hohen Streuquerschnitt auf, so dass es in der Kernindustrie und Kernforschung praktisch unersetzbar ist. In den letzten Jahren hat man versucht, Be in den dem Plasma zugewandten Bauteilen eines Fusionsreaktors einzusetzen, wo es die extremen Bedingungen ertragen soll. ${ }^{[15]}$

Beim Beryllium werden dünne Filme hauptsächlich über die physikalische Gasphasenabscheidung (PVD) erzeugt. ${ }^{[16,117]}$ Beispielsweise hat man Be-Filme auf Kochsalzsubstraten, durch die Verdampfung von Be im Hochvakuum, erzeugt. ${ }^{[118]}$ Das dazu benötigte flüssige Beryllium und sein Dampf wurden als sehr korrosiv beschrieben und die Wahl des Tiegelmaterials war entscheidend, wobei Wolfram empfohlen wurde. Eine weitere Möglichkeit zur Erzeugung von Filmen ist das Sputtern mit einem Ionenstrahl. $\mathrm{Wu}$ und Mitarbeiter 
verwendeten ein Be-Target, um das Wachstum von Be auf $\mathrm{Si}(110)$, und welchen Effekt der Einfluss einer $\mathrm{Cu}$ Dotierung auf den Film hat, zu untersuchen. ${ }^{[19]}$ Eine deutliche Verkleinerung der Partikelgröße und eine Abnahme der Oberflächenrauhigkeit korreliert mit der Zunahme des $\mathrm{Cu}$-Gehalts. Dicke Beschichtungen mit Be $(\sim 150 \mu \mathrm{m})$ werden durch Gleichstrommagnetronsputtern auf sphärischen Polymersubstraten möglich. ${ }^{[120]}$ Die chemische Gasphasenabscheidung ist anderen Methoden zur Erzeugung dünner Filme deutlich überlegen. Über CVD-Prozesse zur Abscheidung von Metallen finden sich viele Bände in Bibliotheken, aber nur wenige über die CVD von Beryllium. Die klassischen CVD-Präkursoren die Halogenide und die Hydride - sind für die Gasphasenabscheidung von $\mathrm{Be}$ ungeeignet, da sie thermisch zu stabil sind und das $\mathrm{BeH}_{2}$ als Polymer, ohne erkennbaren Sublimationsdruck, vorliegt. Einen Ausweg bieten thermisch empfindliche metallorganische Präkursoren in MOCVD-Prozessen. Traditionell sind Chemie und MOCVD-Verfahren eng verzahnt. Zahlreiche neue Verbindungen werden ständig entdeckt, um ihre Eignung als Präkursor zu prüfen, aber beim Beryllium untersuchte man bisher nur ein paar einfache Verbindungen. Nach unserem Kenntnisstand gibt es nur zwei Berichte über metallorganische Verbindungen des Be im Zusammenhang mit MOCVD-Prozessen, darunter das $\mathrm{BeMe}_{2}$, das $\mathrm{BeEt}_{2}$, das $\mathrm{Be}^{t} \mathrm{Bu}_{2}$ und das $\mathrm{BeCp}_{2}$. ${ }^{[21,122]}$ Dieser Mangel an Studien kann auf die erhöhte Gefährdung beim Umgang mit flüchtigen Feststoffen und Flüssigkeiten zurückzuführen sein, die mit bereits geringen Mengen Wasser oder Sauerstoff heftig reagieren und dabei giftigen Staub freisetzen anstatt nur $\mathrm{zu}$ verklumpen. Der Experimentator muss also auch kleinste Leckagen der MOCVD-Kammer vermeiden, um den Eintritt von Luftbestandteilen und den Austritt von BeVerbindungen ausschließen zu können. Die Verwendung von $\mathrm{BeCp}_{2}$ und $\mathrm{BeEt}_{2}$ zur Dotierung von III-VHalbleitern, wie GaAs und GaN, ist dagegen gebräuchlicher. ${ }^{[123,124]}$ Das Beryllium bringt $p$ Dotierungen ein und im Gegensatz $\mathrm{zu} \mathrm{Zn}$ und $\mathrm{Mg}$ verdampft es aufgrund seines kleinen Dampfdrucks nicht aus heißen Reaktorzonen. Weiterhin wird der Dotierungsprozess deutlich reproduzierbarer, da das $\mathrm{Be}$ besser diffundiert. ${ }^{[125]}$ Neben der Abscheidung von metallischen Be-Filmen haben auch noch die MOCVDAbscheidungen von $\mathrm{Be}_{2} \mathrm{C}, \alpha-\mathrm{Be}_{2} \mathrm{~N}_{3}$ und $\mathrm{BeO}$ eine Bedeutung. Bislang wurden solche Beschichtungen nur über PVD-Prozesse hergestellt, so zum Beispiel das $\alpha$ $\mathrm{Be}_{2} \mathrm{~N}_{3}$, welches mit Hilfe der Laserablation von Be-Folie bei $700{ }^{\circ} \mathrm{C}$ unter $\mathrm{N}_{2}$-Atmosphäre auf Silizium aufgebracht wurde. ${ }^{[126]} \mathrm{Be}_{2} \mathrm{C}$ ließ man durch Magnetronsputtern von $\mathrm{Be}$ in $\mathrm{CH}_{4}$-Atmosphäre auf verschiedene Substrate wie $\mathrm{Si}$, Glas, Al-Folie und Polymere aufwachsen. ${ }^{[127]}$ Amorphe BeO-Filme konnte man durch Reaktivsputtern von Be in sauerstoffhaltigem Argon erzeugen. ${ }^{[128]}$ Metallorganische Be-Verbindungen können auch „Single-SourcePräkursoren“ sein, indem der Ligand gleichzeitig N-, Coder O-Atome enthält. Seltene Beispiele sind die Bildung von $\mathrm{Be}_{2} \mathrm{C}$-Filmen unter Verwendung von $\mathrm{BeEt}_{2}$ in Kombination mit $\mathrm{H}_{2}$ als Reaktivgas in plasmaunterstützten CVD-Prozessen (PECVD) bei $250{ }^{\circ} \mathrm{C}^{[129]}$ und der Einsatz von $\mathrm{BeMe}_{2}$ als Vorstufe von ALDVerfahren zur Erzeugung von $\mathrm{BeO}$ auf $\mathrm{Si}$, GaAs ${ }^{[130]}$ und
GaN. ${ }^{[131]}$ Die Verwendung von Be wird stark von der Nuklearindustrie geprägt. Es findet sich eine möglicherweise sehr interessante Anwendung: Urandioxid $\mathrm{UO}_{2}$ ist der am meisten verbreitete Kernbrennstoff, der typischerweise in Form kleiner Pellets eingesetzt wird. Leider schränkt die geringe thermische Leitfähigkeit den Wärmetransport aus dem Pellet heraus stark ein. Um eine Beschädigung der Pellets durch thermische Beanspruchungen zu vermeiden, ist die maximale Betriebstemperatur limitiert. $\mathrm{BeO}$ ist ein ausgezeichneter Wärmeleiter, wiedersteht der starken Strahlung und ist auch bei hohen Temperaturen chemisch inert. Daher erwägt man den Einsatz eines $\mathrm{UO}_{2}-\mathrm{BeO}-$ Kompositmaterials, um die thermische Leitfähigkeit des Kernbrennstoffs zu erhöhen, und so durch Steigerung der Kernbrennstofftemperatur insgesamt die Effizienz des Gesamtprozesses zu vergrößern. ${ }^{[132 \mathrm{a})-\mathrm{c})]}$

Um einen besseren Überblick zu bekommen, führen wir eine Auswahl an Veröffentlichungen in der folgenden Tabelle auf. Diese listet die oben diskutierten Themenfelder und soll als Ankerpunkt weiterer Literaturrecherchen dienen.

\begin{tabular}{|c|c|}
\hline Inhalt $^{[a]}$ & Literatur \\
\hline Toxikologie & {$[2],[5],[6],[10]-[50]$} \\
\hline Sicherheit & $\begin{array}{c}{[6],[10],[12],[29],[30]-} \\
{[36],[48],[49]}\end{array}$ \\
\hline Charakterisierung & [53]-[61],[115]-[131] \\
\hline $\begin{array}{c}\text { Koordinationsverbindungen - } \\
\text { wässriges Milieu }\end{array}$ & {$[3],[65]$} \\
\hline $\begin{array}{c}\text { Koordinationsverbindungen - } \\
\text { wasserfreies Milieu }\end{array}$ & {$[65]-[89]$} \\
\hline $\begin{array}{c}\text { Koordinationsverbindungen - } \\
\text { Ammoniak }\end{array}$ & {$[63],[64]$} \\
\hline $\begin{array}{l}\text { Metallorganische } \\
\text { Verbindungen }\end{array}$ & {$[62],[66],[81],[90]-[100]$} \\
\hline Theoretische Untersuchungen & [62]-[64],[100]-[108] \\
\hline Übersichtsartikel & {$[3],[4],[47],[65],[66],[81]$} \\
\hline
\end{tabular}

[a] (Eine Übersicht zu Artikeln, die einen besonderen Aspekt behandeln)

\section{Und warum sollte nun auch ich mit Beryllium} arbeiten? 
Falls das zuvor Gesagte nicht motivierend genug gewesen sein sollte, um den Aufbau einer eigenen Berylliumchemie anzuregen, fassen wir diesen Bericht mit einer höchstpersönlichen und hoffentlich motivierender Aussage zusammen und schließen mit dieser:

Als Wissenschaftler ist es unser intrinsischer und instinktiver Antrieb, Neues zu entdecken. Für den Chemiker bedeutet das häufig neue Verbindungen und Konzepte zu erschließen, um weiter Licht ins Dunkel des terra incognita chemischer Stoffe zu bringen. Das Streben nach Neuem ist der Stimulus - es rührt von der schieren Freude her die man empfindet, wenn man etwas herausgefunden und verstanden hat. ${ }^{[133]}$ Nur wenige Elemente des Periodensystems wurden von Forschern so stiefmütterlich behandelt wie das Beryllium. Es muss neu entdeckt werden mit all seinen chemischen Eigenschaften und Konzepten! Warten gar neue Substanzklassen und Materialien auf uns? Wir hegen die Hoffnung, dass zukünftig möglichst viele engagierte anorganische Chemiker zum phönix-artigen Aufstieg des Berylliums beitragen, was zu seiner Renaissance führen wird. Solche Wiedergeburten hat es bereits kürzlich gegeben und das an ebenso vernachlässigten Elementen wie dem Uran eindrucksvoll dargelegt in einem hervorragend verfassten Artikel von Stephen Liddle. ${ }^{[134]}$

Immer noch nicht mitreißend genug? Hier ein paar offene Fragen, um auf den schon rollenden Zug, mit dem Chemiker als Führer, aufzuspringen:

\section{Kann man Be(I)-Verbindungen herstellen?}

Gibt es Grignard-artige Reaktionen mit Beryllium?

Was sind die geeigneten Präkursoren für die CVD oder PVD von Beryllium?

Kann Beryllium in seinen Verbindungen $\pi$-Bindungen ausbilden?

\section{Wie kann man Be-Metallbindungen gezielt herstellen?}

Und viele weitere...

Sie sind der Ansicht, dass sie zu einer der obigen Fragen möglicherweise Ideen $\mathrm{zu}$ deren Lösung haben? Dann haben wir unser Hauptziel erreicht: Ihr Interesse geweckt und in Neugier umgewandelt zu haben.

\section{Dank}

DN, GB und SS möchten sich besonders bei Herrn B. Tobey für seine unglaublich kreativen und auch wissenschaftlichen Anregungen während der Arbeit an diesem Manuskript bedanken. MRB und FK danken der DFG für die Förderung der Grundlagenforschung auf dem Gebiet der Berylliumchemie. Weiterhin danken sie Herrn Stefan S. Rudel für seine unfreiwillige Expositionsstudie und der folgenden Evaluation des Notfallteams und der Krankenhausangestellten: Durch Kommunikationsprobleme innerhalb dieses Personenkreises wurden aus $250 \mathrm{mg}$ Berylliumchlorid $250 \mathrm{~g}$ Beliriumfluorid (sic!). Es empfiehlt sich daher immer einen verunfallten Kammeraden ins Krankenhaus zu begleiten. Wir danken Herrn Dr. Roland Paul (Bundesanstalt für Arbeitsschutz und Arbeitsmedizin, Berlin) für die Zahlen zur Be-Exposition und Herrn Dr. (RUS) Sergei Ivlev für die Recherche nach und die Übersetzung der russischen Sicherheitsvorschriften ins Deutsche. Wir danken den edlen Spendern von Berylliummetall (u.a. Prof. Arndt Simon, Stuttgart) und von alten Röntgenröhren.

[1] a) R. Höber, F. Kiesow, Zeitschr. f. physikal. Chemie 1898, 27 (4), 601-616 b) R. Höber, F. Kiesow, Zeitschrift für Psychologie und Physiologie der Sinnesorgane 1900, 23.

[2] O. Kumberger, H. Schmidbaur, Chemie in unserer Zeit. 1993, 27 (Nr. 6), 310-316.

[3] a) H. Schmidbaur, Coord. Chem. Rev. 2001, 215, 223-242. b) $2000-2016: 1559$ articles on beryllium chemistry thereof 1040 involving computational methods (Web Of Science 07.04.2016)

[4] a) K. A. Walsh in Beryllium Chemistry and Processing (Eds.: E. E. Vidal, A. Goldberg, E. N. C. Dalder, D. L. Olson, B. Mishra), ASM International, 2009, vol. 1. b) https://www.nasa.gov/press-release/nasas-james-webbspace-telescope-primary-mirror-fully-assembled

[5] Deutsche Gesetzliche Unfallversicherung, Berufskrankheiten-Geschehen der Deutschen Gesetzlichen Unfallversicherung, http://www.dguv.de/de/Zahlen-undFakten/BK-Geschehen/index.jsp, abgerufen am 11.02.2015.

[6] Deutsche Gesetzliche Unfallversicherung, DGUV-Statistik für die Praxis 2013, 2014.

[7] Institute of Occupational Medicine: IOM, SHEcan Report P937/4, Health, socio-economic and environmental aspects of possible amendments to the EU Directive on the protection of workers from the risks related to exposure to carcinogens and mutagens at work Beryllium and beryllium compounds $\mathbf{2 0 1 1}$.

[8] Bundesanstalt für Arbeitsschutz und Arbeitsmedizin Nationales Asbest-Profil Deutschland 2015, www.baua.de/dok/6549238.

[9] A. Seidle, Gmelin Handbook of Inorganic Chemistry; Be Beryllium. Suppl. Vol. A 1: The Element, Springer-Verlag. Berlin-Heidelberg-New York-Tokyo, 1986, 8, 300 - 315.

[10] H. Zorn \& G. Fischer, $I V$ - 2.2; Leichtmetalle; $I V$ - 2.2.5; Beryllium; Handbuch der Arbeitsmedizin, ecomed Medizin: Landsberg, 1989.

[11] J. R. Bill, D. G. Mack, M. T. Falta, L. A. Maier, A. K Sullivan, F. G. Joslin, A. K. Martin, B. M. Freed, B. L. Kotzin \& A. P. Fontenot, J. Immunol., 2005, 175, 70297037.

[12] B. Schaller, K. H. Schaller \& H. D. Drexler, II-1.1.B-2; Beryllium; Handbuch der Arbeitsmedizin, ecomed Medizin: Landsberg, 2008, 7 Erg.Lfg. 5/08.

[13] K. J. Cummings, A. B. Stefaniak, M. A. Virji \& K. Kreiss, Environ. Health Perspect., 2009, 117, 1250 - 1256.

[14] A. B. Stefaniak, Ann. Occup. Hyg., 2011, 55, 556-557.

[15] a) C. Strupp, Ann. Occup. Hyg., 2011, 55, 43-56; b) C. Strupp, Ann. Occup. Hyg., 2011, 55, 30-42; c) C. Strupp, Ann. Occup. Hyg., 2011, 55, 558-559.

[16] P. Boffetta, J. P. Fryzek \& J. S. Mandel, Crit. Rev. Toxicol., 2012, 42, 107-118.

[17] C. R. Schuler, M. A. Virji, D. C. Deubner, M. L. Stanton, A. B. Stefaniak, G. A. Day, J. Y. Park, M. S. Kent, R. Sparks \& K. Kathleen, Scand. J. Work Environ. Health, 2012, 38, $270-281$ 
[18] G. Clayton, Y. Wang, F. Crawford, A. Novikov, B. Wimberly, J. Kieft, M. Falta, N. Bowerman, P. Marrack, A. Fontenot, S. Dai, J. Kappler, Cell, 2014, 158, 132-142]

[19] J. Ding, L. Lin, W. Hang \& X. Yan, Metallomics, 2009, 1, 471-478.

[20] L. S. Newman, Science, 1993, 262, 197-198.

[21] M. Schwenk, R. Klein \& D. M. Templeton, Pure Appl. Chem., 2008, 80, 1349 - 1364.

[22] A. P. Fontenot, T. S. Keizer, M. McCleskey, D. G. Mack, R. Meza-Romero, J. Huan, D. M. Edwards, Y. K. Chou, A. A. Vandenbark, B. Scott \& G. G. Burrows, J Immunol., 2006, 177, 3874-3883.

[23] N. A. Bowerman, M. T. Falta, D. G. Mack, J. W. Kappler \& A. P. Fontenot, J. Immunol., 2011, 187, 3694-3703.

[24] Regulation (EC) No 1272/2008, Official Journal of the European Union 2008, L353, 1 - 1355

[25] B. L. Scott, T. M. McCleskey, A. Chaudhary, E. HongGeller \& S. Gnanakaran, Chem. Commun., 2008, 2837 2847.

[26] T. McCleskey, D. Ehler, T. Keizer, D. Asthagiri, L. Pratt, R. Michalczyk \& B. Scott, Angew. Chem., 2007, 119 , 2723-2725

[27] B. L. Scott, Z. Wang, B. L. Marrone \& N. N. Sauer, J. Inorg. Biochem., 2003, 94, 5-13.

[28] D. Kriebel, J. D. Brain, N. L. Sprince, H. Kazemi, American Review of Respiratory Disease, 1988, 137, 464 473

[29] Deutsche Gesetzliche Unfallversicherung, Handlungsanleitung für die arbeitsmedizinische Vorsorge BGI/GUV-I 504-40c, März 2009 nach dem Berufsgenossenschaftlichen Grundsatz G40 "Krebserzeugende und erbgutverändernde Stoffe allgemein, hier: Beryllium", http://publikationen.dguv.de/dguv/pdf/10002/i-50440c.pdf, abgerufen am 25.08.2015.

[30] a) http://gestis.itrust.de, search entry: „beryllium”, [07985], 25.8.2015; b) IPCS: CICADs - Concise International Chemical Assessment Documents, WHO, Genf, since 1998.

[31] a) http://gestis.itrust.de, search entry: ,beryllium”, [07619], 25.8.2015; b) Hartwig, Andrea (Hrsg.), Gesundheitsschädliche Arbeitsstoffe DFG: Toxikologischarbeitsmedizinische Begründungen von MAK-Werten; Wiley-VCH, 2015, 59.Lieferung.

[32] http://gestis.itrust.de, search entry: „,beryllium”, [07510], 25.8.2015.

[33] http://gestis.itrust.de, search entry: „,beryllium”, [99983], 25.8.2015.

[34] a) http://gestis.itrust.de, search entry: ,beryllium”; b) §4 ArbMedVV, Verordnung zur Arbeitsmedizinischen Vorsorge, Bundesrecht; c) I. Brüske, E. Thiering, J. Heinrich, H.-E. Wichmann, K. Huster, D. Nowak „Chronisch-obstruktive Atemwegserkrankung (COPD) durch Inhalation von anorganischen Stäuben am Arbeitsplatz" Forschungsbericht Arbeitsschutz 428, BMAS, 15.11.2012

[35] Technische Regeln für Gefahrstoffe (TRGS) 410, Ausgabe Juni 2015 GMBl 2015, S. 587-595 [Nr. 30] (v.5.8.2015), Bundesanstalt für Arbeitsschutz und Arbeitsmedizin (baua).

[36] http://www.dguv.de/de/Pr\%C3\%A4vention/Themen-AZ/Gefahrstoffe/index.jsp, „Krebserzeugende Metalle” M. Wieske, 14.10.2014.

[37] R. Machloz, H.-J. Lewerenz in Lebensmitteltoxikologie, Springer Verlag Berlin, Heidelberg, 1989.
[38] A. Widener, $C \& E N$, 2015, Vol.93, 32, 5.

[39] Title 10, Code of Federal Regulations, "Energy", Chapter 3, "Department of Energy", Part 850, "Chronic Beryllium Disease Prevention Program", http://www.ecfr.gov/cgibin/textidx?SID=a1472d963ff100 $653609784421 \mathrm{dc} 6 \mathrm{c} 05 \& \mathrm{mc}=$ true $\&$ node $=$ pt10.4.850\& $\mathrm{rgn}=$ $\operatorname{div} 5$.

[40] Title 29, Code of Federal Regulations, "Labor", Chapter 17, "Occupational Safety and Health Administration, Department of Labor", Part 1910, "Occupational Safety and Health Standards", Subpart H, "Hazardous Materials", Section 120, "Hazardous Waste Operations and Emergency "Hesponse", https://www.osha.gov/pls/oshaweb/owadisp.show_docum ent?p_table $=$ STANDARDS\&p_id $=9765$.

[41] Title 29, Code of Federal Regulations, "Labor", Chapter 17, "Occupational Safety and Health Administration, Department of Labor", Part 1910, "Occupational Safety and Health Standards", Subpart I, "Personal Protective Equipment", Section 132, "General Requirements", https://www.osha.gov/pls/oshaweb/owadisp.show docum ent?p_table $=$ STANDARDS\&p_id $=9777$.

[42] Title 29, Code of Federal Regulations, "Labor", Chapter 17, "Occupational Safety and Health Administration, Department of Labor", Part 1910, "Occupational Safety and Health Standards", Subpart Z, "Toxic and Hazardous Substances", Section 1200, "Hazard Communication", https://www.osha.gov/pls/oshaweb/owadisp.show_docum ent?p_table=STANDARDS\&p_id=10099.

[43] Chronic Beryllium Disease Prevention Program, SLAC National Accelerator Laboratory 2016, https://wwwgroup.slac.stanford.edu/esh/documents/beryllium.pdf.

[44] Health and Safety Executive: Beryllium and you, http://www.hse.gov.uk/pubns/indg311.pdf

[45] ГОСТ 12.1 005-76. Воздух рабочей зоны. Общие санитарно-гигиенические требования. - М.: 1976.

[46] Б.Н. Колбасов, Д.К. Курбатов. Обеспечение безопасности при обращении с бериллием и его отходами на площадке термоядерной установки. // Вопросы атомной науки и техники. Cер. Термоядерный синтез. - 2003. - Вып. 3. - С. 32-60

[47] G. Brauer (Hrsg.), Handbook of Preparative Inorganic Chemistry. 2. Auflage., Academic Press, 1963, vol. 1.

[48] Deutsche Gesetzliche Unfallversicherung, Handlungsanleitung für die arbeitsmedizinische Vorsorge Dezember 2011 BGR/GUV-R 190, „Benutzung von Atemschutzgeräten", http://publikationen.dguv.de/dguv/pdf /10002/r-190.pdf, abgerufen am 25.08.2015.

[49] Deutsche Gesetzliche Unfallversicherung, Oktober 2010 BGI/GUV-I 504-26, Handlungsanleitung für die arbeitsmedizinische Vorsorge nach dem DGUV Grundsatz G26 "Atemschutzgeräte“, http://publikationen.dguv.de/dguv/pdf/10002/i-504-26.pdf, abgerufen am 25.08.2015.

[50] H. Schmidbaur, Gmelin Handbook of Inorganic Chemistry-Be. Organoberyllium Compounds, Springer Verlag Berlin, 1987.

[51] The manipulation of air-sensitive compounds, 2nd edition, D. F. Shriver, M. A. Drezdzon, 1986 John Wiley\& Sons, Inc.

[52] O. Hönigschmid, T. Johannsen, Z. Naturforsch. 1946, 1, 650-655.

[53] http://chem.ch.huji.ac.il/nmr/techniques/1d/row2/be.html, 25.8.2015

[54] R. A. Kovar, G. L. Morgan, J. Am. Chem. Soc. 1970, 92, 5067-5072. 
[55] D. F. Gaines, K. M. Coleson, D. F. Hillenbrand, Journal of Magnetic Resonance 1981, 4, 84-88.

[56] K. W. Nugent, J. K. Beattie, L. D. Field, J. Phys. Chem. 1989, 93 (14), 5371-5377.

[57] I. Hung, C. L. B. Macdonald, R. W. Schurko, Chem. Eur. J. 2004, 10, 5923-5935.

[58] P. G. Plieger, K. D. John, T. S. Keizer, T. M. McCleskey, A. K. Burell, R. L. Martin, J. Am. Chem. Soc. 2004, 126 , 14651-14658.

[59] R. E. Dinnebier, S. J. L. Billinge, Powder Diffraction: Theory and Practice, RCS Publishing, 2008.

[60] X-ray Photoelectron Spectroscopy Database, Version 4.1 (National Institute of Standards and Technology, Gaithersburg, 2012); http://srdata.nist.gov/xps/.

[61] P. Echlin, Handbook of Sample Preparation for Scanning Electron Microscopy and X-Ray Microanalysis, Springer, 2009.

[62] D. Naglav, B. Tobey, A. Neumann, D. Bläser, C. Wölper, S. Schulz, Organometallics 2015, 34 (12), 3072-3078.

[63] F. Kraus, S. A. Baerl, M. R. Buchner, A. J. Karttunen, Chem. Eur. Jour. 2012, 18 (7), 2131-2142.

[64] F. Kraus, S. A. Baerl, M. Hoelzl, A. J. Karttunen, Eur. J. Inorg. Chem. 2013, 24, 4184-4190.

[65] K. Dehnicke, B. Neumüller, Z. Anorg. Allg. Chem. 2008, 634, 2703-2728.

[66] K. J. Iversen, S. A. Couchman, D. J. D. Wilson, J. L. Dutton, Coord. Chem. Rev. 2015, 297-298, 40-48.

[67] H. Bürger, C. Forker, G. Goubeau, Mh. Chem. 1965, 96, 597-601.

[68] A. H. Clark, A. Haaland, Chem. Commun. 1969, 912-913.

[69] A. H. Clark, A. Haaland, Acta Chemica Scandinavia 1970, 24, 3024-3030.

[70] . L. Atwood, G. D. Stucky, Chem. Commun. 1967, 11691170 .

[71] H. Nöth, D. Schlosser, Inorg. Chem. 1983, 22, 2700-2703.

[72] R. A. Andersen, G. E. Coates, J. C. S. Dalton 1972, 21532155.

[73] W. A. Herrmann, O. Runte, G. Artus, J. Organomet. Chem. 1995, C1-C4, 501.

[74] R. Han, G. Parkin, Inorg. Chem. 1992, 31, 983-988.

[75] R. Han, G. Parkin, Inorg. Chem. 1993, 32, 4968-4870.

[76] Y. Sohrin, H. Kokusen, S. Kihara, M. Matsui, Y. Kushi, M. Shiros, J. Am. Chem. Soc. 1993, 115, 4128-4136.

[77] R. Fleischer, D. Stalke, Inorg. Chem. 1997, 36, $2413-$ 2419.

[78] H. Nöth, D. Schlosser, Eur. J. Inorg. Chem. 2003, 22452254.

[79] M. P. Dressel, S. Nogai, R. J. F. berger, H. Schmidbaur, $Z$. Naturforsch. 2003, 58b, 173-182.

[80] S. C. Chmely, T. P. Hanusa, W. W. Brennessel, Angew. Chem. Int. Ed. 2010, 49, 5870-5874.

[81] M. S. Hill, Inorg. Chem. 2013, 109, 18-27.

[82] M. Arrowsmith, M. S. Hill, G. Kociok-Köhn, D. J. MacDougall, M. F. Mahon, Angew. Chem. Int. Ed. 2012 $51,2098-2100$

[83] J. Gottfriedsen, S. Blaurock, Organometallics 2006, 25, 3784-3786.
[84] R. J. Gilliard Jr., M. Y. Abraham, Y. Wang, P. Wei, Y. Xie, B. Quillian, H. F. Schaefer III, P. v. R. Schleyer, G. H. Robinson, J. Am. Chem. Soc., 2012, 134 (24), $9953-$ 9955.

[85] M. Arrowsmith, M. S. Hill, G. Kociok-Köhn, D. J MacDougall, M. F. Mahon, I. Malloy, Inorg. Chem. 2012, 52 (24), 13408-13418.

[86] M. Arrowsmith, M. R. Crimmin, M. S. Hill, G. KociokKöhn, Dalton Trans. 2013, 42, 9720-9726.

[87] T. Arnold, H. Braunschweig, W. C. Ewing, T. Kramer, J. Mies, J. K. Schuster, Chem. Commun. 2015, 51, 737-740.

[88] a) D. Naglav, D. Bläser, C. Wölper, S. Schulz, Inorg. Chem. 2014, 53 (2), 1241-1249. b) D. Naglav, B. Tobey, C. Wölper, D. Bläser, G. Jansen, S. Schulz, Eur. J. Inorg. Chem. 2016, accepted for publication.

[89] D. Naglav, A. Neumann, D. Bläser, C. Wölper, R. Haack, G. Jansen, S. Schulz, Chem. Commun. 2015, 51, 38893891.

[90] H. Gilman, F. Schulze, J. Chem. Soc., 1927, 2663-2669.

[91] A. I. Snow, R. E. Rundle, Acta. Cryst.1951, 4, 348.

[92] G. E. Coates, F. Glockling, N. D. Huck, J. Chem. Soc. 1952, 4496-4501.

[93] G. E. Coates, F. Glockling, J. Chem. Soc. 1954, 22-27.

[94] A. Almenningen, A. Haaland, J. E. Nilsson, Acta Chemica Scandinavia 1968, 22, 972-976.

[95] G. E. Coates, R. C. Srivastava, J. Chem. Soc. 1972, 15411544.

[96] D. Seyerferth, Annual Survey Covering The Year 1972, Department of Chemistry MIT, 19-24.

[97] K. Ruhlandt-Senge, R. A. Bartlett, M. M. Olmstead, P. P. Power, Inorg. Chem. 1993, 32, 1724-1728.

[98] M. Niemeyer, P. P. Power, Inorg Chem. 1997, 36, 46884696.

[99] D. Himmel, H. Scherer, D. Kratzert, I. Krossing, Z. Anorg. Allg. Chem. 2015, 6418 (3-4), 655-659.

[100] R. Fernández, E. Carmona, Eur. J. Inorg. Chem. 2005, 3197-3206.

[101] Y. Xie, H. F. Schaefer, E. D. Jemmis, Chemical Physics Letters 2005, 402, 414-421.

[102] A. Velazquez, I. Fernández, G. Frenking, G. Merino, Organometallics 2007, 26, 4731-4736.

[103] S. Duley, P. Goyal, S. Giri, P. K. Chattaraj, Croat. Chem. Acta 2009, 82 (1), 193-205.

[104] M. H. Al-Mokhtar, L. O. Mó, M. Yáñez, J.-C. Guillemin, Dalton Trans. 2010, 39, 4593-4601.

[105] S. De, P. Parameswaran, Dalton Trans. 2013, 42, 46504656.

[106] S. A. Couchman, N. Holzmann, G. Frenking, D. J. D. Wilson, J. L. Dutton, Dalton Trans 2013, 42, 11375 11384 .

[107] K. J. Iversen, D. J. D. Wilson, J. L. Dutton, Organometallics 2013, 32 (21), 6209-6217.

[108] I. Alkorta, J. Elguero, A. Mó, M. Yáñez, J.E. Del-Bene, Phys. Chem. Chem. Phys. 2015, 17, 2259-2267.

[109] S. Pan, R. Saha, P. K. Chattaraj, New J. Chem. 2015, 39, 6778-6786.

[110] S. Borocci, N. Bronzolino, F. Grandinetti, Chem. Phys. Lett. 2005, 406, 179-183. 
[111] B. G. S. Brito, G.-Q. Hai, L. Cândido, Chem. Phys. Lett. 2013, 586, 108-110.

[112] M. Šulka, M. Pitoňák, I. Černušák, M. Urban, P. Neogrády, Chem. Phys. Lett. 2013, 573, 8-14.

[113] O. Brea, O. Mó, M. Yáñez, I. Alkorta, J. Elguero, Chem. Eur. J. 2015.

[114] M. Yáñez, O. Mó, I. Alkorta, J. Elguero, Chem. Eur. J. 2013, 19, 11637-11643.

[115] M. Merola et al., Fusion Engineering and Design 2010, 2312-2322.

[116] R. O. Adams, C. W. Nordin, Thin Solid Films 1989, $375-$ 381.

[117] A. R. Berry, C. W. Nordin, Thin Solid Films 1992, 83-86.

[118] B. J. Mulder, Thin Solid Films 1978, 35-40.

[119] M-J. Zhou et al., Trans. Nonferrous Met. Soc. China 2012, $1151-1155$

[120] H. Xu et al., Thick Beryllium Coatings by Magnetron Sputtering. MRS Proceedings 2011, 1339-1345.

[121] J. M. Wood, F. W. Frey, Chemical Vapour Deposition of Beryllium Metal, Proceedings of the conference on chemical vapour deposition of refractory metals, alloys and compounds, 1967, 205-217

[122] K. V. Salazar, S. G. Pattillo, M.Trkula, Fusion Science and Technology 2000, 69-73.

[123] J. D. Parsons, L. S. Lichtmann, F. G. Krajenbrink, D. W. Brown, J. Cryst. Growth 1986, 32-36.

[124] D. Suzuki et al., International Conference on Indium Phosphide and Related Material 1997, 540-543

[125] T. Kobyashi, K. Kurishima, T. Ishibashi, J. Cryst. Growth 1994, 1-4.

[126] G. Soto, J.A.Díaz, R.Machorro, A. Reyes-Serrato, W. de la Cruz, Mat. Let. 2002, 29-33.

[127] Xie et al, Fusion Technology 2000, 384-390.

[128] D. Schalch, A. Scharman, A. Weiß, Thin Solid Films 1985, 351-358.

[129] W-S. Shih, R. B. Stephens, W. J. James, Fusion Science and Technology 2000, 24-31.

[130] J. H. Yum et al., J. Crys. Growth 2011, 126-133.

[131] D. W. Johnson, J. Electron. Mater. 2014, 151-154.

[132] a) S.Ishimotoi, M. Hirai, K. Itot, Y. Korei,J. Nucl. Sci. Technol.33, 2, 1996, 134-140. b) W. Zhou, R. Liu, S. T. Revankar, Ann. Nucl. Energy, 81, 2015, 240-248. c) R.Lattaa, S. T. Revankara, A. A. Solomona, Heat Transfer Eng.29, 4, 2008, 357-365.

[133] The Pleasure of Finding Things out. The Best Short Works of Richard P. Feynman. Penguin, 2001.

[134] S. Liddle, Angew. Chem. Int. Ed. 2015, 54, 8604-8641.

Received: ((will be filled in by the editorial staff)) Published online: ((will be filled in by the editorial staff)) 


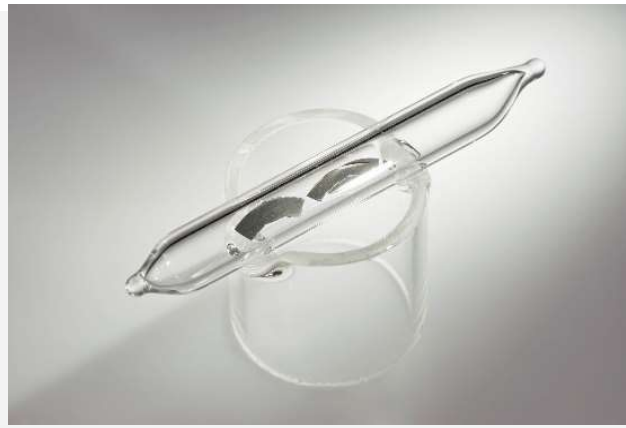

D. Naglav, M. R. Buchner, G. Bendt, F. Kraus*, S. Schulz*........ Page 1. - Page 14.

Auf neuen Pfaden - Per Anhalter durch die Berylliumchemie 


\section{DuEPublico}

This text is made available via DuEPublico, the institutional repository of the University of Duisburg-Essen. This version may eventually differ from another version distributed by a commercial publisher.

DOI: $\quad 10.1002 /$ ange.201601809

URN: urn:nbn:de:hbz:464-20201203-171510-5

This is the peer reviewed version of the following article: Angew. Chem. 2016,128, 10718-10733, which has been published in final form at:

https://doi.org/10.1002/ange.201601809

All rights reserved. 